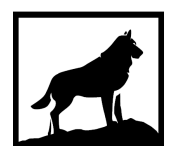

Michigan Technological

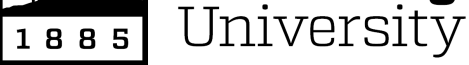

Michigan Technological University Digital Commons @ Michigan Tech

\title{
A HIGH ACCURACY MINIMALLY INVASIVE REGULARIZATION TECHNIQUE FOR NAVIER-STOKES EQUATIONS AT HIGH REYNOLDS NUMBER
}

Mustafa Aggul

Michigan Technological University, maggul@mtu.edu

Copyright 2016 Mustafa Aggul

Recommended Citation

Aggul, Mustafa, "A HIGH ACCURACY MINIMALLY INVASIVE REGULARIZATION TECHNIQUE FOR NAVIERSTOKES EQUATIONS AT HIGH REYNOLDS NUMBER", Open Access Master's Thesis, Michigan Technological University, 2016.

https://doi.org/10.37099/mtu.dc.etdr/147

Follow this and additional works at: https://digitalcommons.mtu.edu/etdr

Part of the Numerical Analysis and Computation Commons, and the Partial Differential Equations Commons 


\title{
A HIGH ACCURACY MINIMALLY INVASIVE REGULARIZATION TECHNIQUE FOR NAVIER-STOKES EQUATIONS AT HIGH REYNOLDS NUMBER
}

By

Mustafa Aggul

\begin{abstract}
A THESIS
Submitted in partial fulfillment of the requirements for the degree of MASTER OF SCIENCE

In Mathematical Sciences
\end{abstract}

MICHIGAN TECHNOLOGICAL UNIVERSITY

2016

(C) 2016 Mustafa Aggul 
This thesis has been approved in partial fulfillment of the requirements for the Degree of MASTER OF SCIENCE in Mathematical Sciences.

Department of Mathematical Sciences

Thesis Advisor: Dr. Alexander Labovsky

Committee Member: Dr. Franz X. Tanner

Committee Member: Dr. Aleksandr V. Sergeyev

Department Chair: Dr. Mark S. Gockenbach 


\section{Dedication}

To my mother and the future mother of my kids... 


\section{Contents}

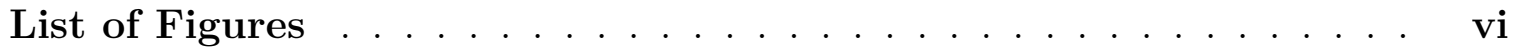

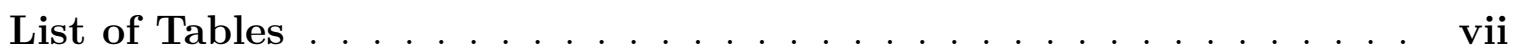

Acknowledgements $\ldots \ldots \ldots \ldots \ldots$ viii

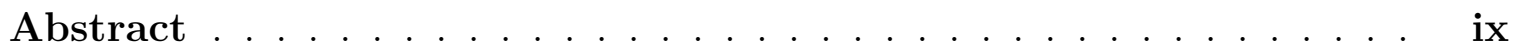

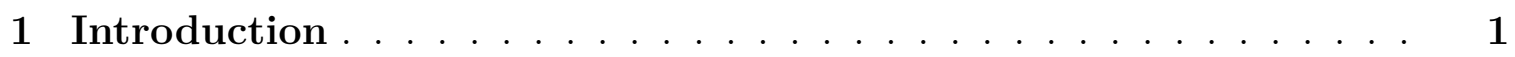

2 Mathematical Preliminaries and Notations . . . . . . . . . 5

3 AV Approximation $\ldots \ldots \ldots \ldots \ldots \ldots \ldots \ldots$

3.1 Stokes Projection . . . . . . . . . . . . . . . . 11

3.2 Stability of the AV approximation . . . . . . . . . . . 11

3.3 Error Estimates of AV Approximation . . . . . . . . . . . . . 12

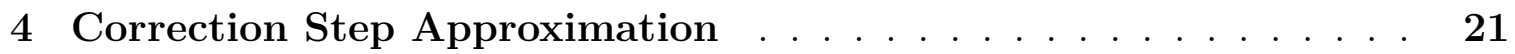

4.1 Stability of the CS Approximation . . . . . . . . . . . . . 21

4.2 Error Estimate of CS Approximation . . . . . . . . . . . 25

5 Computational Tests $\ldots \ldots \ldots \ldots \ldots$

5.1 Quantitative Test . . . . . . . . . . . . . . . . . 31

5.2 Qualitative Test . . . . . . . . . . . . . . . . 34

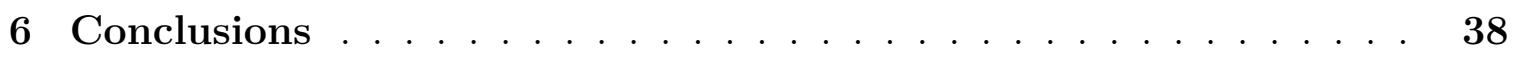




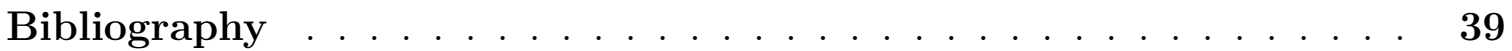




\section{List of Figures}

$5.1 \quad$ DNS velocity field $u \ldots \ldots \ldots \ldots \ldots \ldots \ldots$

5.2 AV Approximation $u_{1}^{h} \ldots \ldots \ldots \ldots \ldots \ldots \ldots$

5.3 AV Approximation zoomed in . . . . . . . . . . . . 35

5.4 CS Approximation $u_{2}^{h} \ldots \ldots \ldots \ldots \ldots \ldots \ldots \ldots \ldots$

5.5 CS Approximation Zoomed in . . . . . . . . . . . . . 36 


\section{List of Tables}

$5.1 \quad$ AV approximation, $\nu=0.01 \ldots \ldots \ldots \ldots \ldots \ldots \ldots$

5.2 Correction step approximation, $\nu=0.01 \ldots \ldots \ldots \ldots \ldots$

5.3 AV approximation, $\nu=0.0005 \ldots \ldots \ldots \ldots \ldots \ldots$

5.4 Correction step approximation, $\nu=0.0005 \ldots \ldots \ldots \ldots \ldots$ 


\section{Acknowledgements}

I would like to thank my advisor Alexander Labovsky for his invaluable help and contribution in my personal development. I would not be the same if I had missed my change to meet him.

I also would like to thank Franz X. Tanner and Aleksandr V. Sergeyev for serving as committee members in my Masters defense. My thesis now looks much better with your corrections and contributions.

My mom! You are the reason of my existence. The feeling that you always stand with me makes me stronger. You have been a great father though by not letting me feel his absence.

Dad! I know you are watching me from somewhere out of this world. No matter where you are now, my heart will always be with you. May you rest in peace!

Love of my life, Deniz Yener. You have always been with me and supporting me with your existence. As we are on the verge of our marriage, my hope is to live happily ever all together.

All the other contributors, my professors, friends, family members, and those whom I could not mention here! Thanks a lot! Hope you always encounter with people like yourselves. 


\section{Abstract}

A method in this work, that combines the defect and deferred correction approaches to approximate solutions of Navier-Stokes equations at high Reynolds number is presented. This method is of high accuracy in both space and time, and it allows for the usage of legacy codes - a frequent requirement in the simulation of turbulent flows in complex geometries. The two-step method is considered here: in order to obtain a regularization, that is second order accurate in space and time, the method computes a low-order accurate, stable and computationally inexpensive approximation (Backward Euler with artificial viscosity) twice. The results are readily extendable to the higher order accuracy cases by adding more correction steps. Both the theoretical results and the numerical tests are provided to demonstrate that the computed solution is stable and the accuracy in both space and time is improved after the correction step. We also perform a qualitative test to demonstrate that the method is capable of capturing qualitative features of a turbulent flow, even on a very coarse mesh. 


\section{Chapter 1}

\section{Introduction}

The motion of incompressible fluid flow in the flow domain $\Omega=(0, L)^{d}$ is governed by the Navier-Stokes equations: find the velocity-pressure pair $u: \Omega \times(0, T] \rightarrow R^{d},(d=$ $2,3)$ and $p: \Omega \times(0, T] \rightarrow R$ satisfying

$$
\begin{aligned}
& u_{t}+u \cdot \nabla u-\nu \Delta u+\nabla p=f, \text { for } x \in \Omega, 0<t \leq T \\
& \nabla \cdot u=0, x \in \Omega, \text { for } 0 \leq t \leq T, \\
& u(x, 0)=u_{0}(x), \text { for } x \in \Omega,
\end{aligned}
$$

with the normalization condition $\int_{\Omega} p(x, t) d x=0$ for $0<t \leq T$. Throughout this paper, we consider the case of homogeneous Dirichlet boundary conditions to simplify the proofs; non-homogeneous Dirichlet boundary conditions can be treated in exactly the same manner and the same results hold.

According to the Kolmogorov theory [24], there exists a continuum of scales in turbulent fluid flow, with the smallest scales (in the case of a $3-D$ flow) being of the order $O\left(R e^{-3 / 4}\right)$, where the Reynolds number $R e$ is inverse proportional to the viscosity coefficient $\nu$. Thus, capturing all the small structures in a turbulent flow requires the number of mesh points in space for each time step to be $O\left(R e^{9 / 4}\right)$ for 
three-dimensional problems. It is not uncommon to have $R e \sim O\left(10^{8}\right)$ in real-life applications.

Hence, the direct numerical simulation (DNS) of a $3-D$ turbulent flow is often not computationally economical or even feasible. Sometimes it is desirable (especially for turbulent flows in complex geometries) to be able to use pre-existing codes. Thus, we are aiming at constructing a method that would approximate a flow at high Reynolds number, while being computationally attractive, stable and of high accuracy in both space and time.

To that end, we consider a defect correction approach from [15]. Defect correction strategies have been successfully applied to stiff systems $[3,16,11,6,18]$, and in particular to evolutionary Navier Stokes Equation (NSE) - see, e.g., [15] and references therein.

The general idea of any Defect Correction Method (DCM) can be formulated as follows (see, e.g., $[27,1])$ :

Find a unique solution of $F x=0$, by

DCM: Use an approximation $\tilde{F}$ to build an iterative procedure:

$$
\begin{gathered}
\tilde{F} x_{1}=0, \\
x_{i+1}=\left(I-\tilde{F}^{-1} F\right) x_{i}, i \geq 1 .
\end{gathered}
$$

The choice of a particular approximation $\tilde{F}$ determines the defect correction method in use. As a result of using the artificial viscosity approximation-based defect correction method of [15], we have an approach that allows for the usage of legacy codes and gives a second order accurate in space approximation of a flow at high Reynolds number. This is obtained by computing two consecutive approximations $u_{1}$ and $u_{2}$ with Backward Euler method and with exactly the same matrix (the correction step 
only modifies the right hand side of the system for $u_{2}$ by a function of the previously computed $u_{1}$ ). These approximations, however, are first order accurate in time. The question is: can we increase the time accuracy without increasing the computational cost? The answer lies in the temporal counterpart of the defect correction idea, known as deferred correction.

The main advantage of the deferred correction approach is that a simple low-order method can be employed, and the recovered solution is of high-order accuracy, due to a sequence of deferred correction equations. The classical deferred correction approach could be seen, e.g., in [28]. However, in 2000 a modification of the classical deferred correction approach was introduced by Dutt, Greengard and Rokhlin, [25]. This allowed the construction of stable and high-order accurate spectral deferred correction methods. In [20], M.L. Minion discusses these spectral deferred correction (SDC) methods in application to an initial value ODE

$$
\begin{aligned}
\phi^{\prime}(t) & =F(t, \phi(t)), t \in[a, b] \\
\phi(a) & =\phi_{a} .
\end{aligned}
$$

The solution is written in terms of the Picard integral equation; a polynomial is used to interpolate the subintegrand function and the obtained integral term is replaced by its quadrature approximation. The deferred correction approach was used to improve the temporal accuracy of a turbulence model in [31].

When both the defect and deferred correction are combined into one method, we seek two approximations $u_{1}^{h, i}$ and $u_{2}^{h, i}$ to the true solution $u\left(t_{i}\right)$. Both are computed with the same matrix of the system, but with different right hand sides. The computational attractiveness is due to two important factors. First, the cost of computing each approximation is the cost of solving a Backward Euler method for the NSE with increased viscosity coefficient - a method which is hard to beat in terms of computational cost. Secondly, the defect-deferred correction methods are readily parallelizable, as the solutions $u_{1}^{h, i+k}, u_{2}^{h, i+k-1}, \ldots, u_{k+1}^{h, i}$ can be computed simultaneously on $k+1$ 
cores to produce a potentially $(k+1)$-order accurate approximation.

We propose the following two-step method that produces a sequence of approximations $\left(u_{1}^{h}, p_{1}^{h}\right),\left(u_{2}^{h}, p_{2}^{h}\right)$ of the true solution $(u, p)$.

$$
\begin{array}{r}
\left(\frac{u_{1}^{h, n+1}-u_{1}^{h, n}}{k}, v^{h}\right)+(h+\nu)\left(\nabla u_{1}^{h, n+1}, \nabla v^{h}\right)+b^{*}\left(u_{1}^{h, n+1}, u_{1}^{h, n+1}, v^{h}\right) \\
-\left(p_{1}^{h, n+1}, \nabla \cdot v^{h}\right)=\left(f\left(t_{n+1}\right), v^{h}\right), \\
\left(\frac{u_{2}^{h, n+1}-u_{2}^{h, n}}{k}, v^{h}\right)+(h+\nu)\left(\nabla u_{2}^{h, n+1}, \nabla v^{h}\right)+b^{*}\left(u_{2}^{h, n+1}, u_{2}^{h, n+1}, v^{h}\right) \\
-\left(p_{2}^{h, n+1}, \nabla \cdot v^{h}\right)=\left(\frac{f\left(t_{n+1}\right)+f\left(t_{n}\right)}{2}, v^{h}\right)+\frac{\nu}{2} k\left(\nabla\left(\frac{u_{1}^{h, n+1}-u_{1}^{h, n}}{k}\right), v^{h}\right) \\
+\frac{1}{2} b^{*}\left(u_{1}^{h, n+1}, u_{1}^{h, n+1}, v^{h}\right)-\frac{1}{2} b^{*}\left(u_{1}^{h, n}, u_{1}^{h, n}, v^{h}\right)+h\left(\nabla u_{1}^{h, n+1}, \nabla v^{h}\right),
\end{array}
$$

where $b^{*}(\cdot, \cdot, \cdot)$ is the explicitly skew-symmetrized trilinear form, defined below.

The remainder of this paper is organized as follows. Section 2 introduces the necessary notation and preliminaries; then follows Section 3 on the accuracy and stability of the defect step approximation. These results come mostly from [15], as the equation for the defect step of the defect-deferred approach is exactly the defect step of the approach in [15]. The novelty of the proposed method appears in Section 4, where stability and increased accuracy (both time and space) of the correction step is studied. The quantitative and qualitative computational tests are presented in Section 5 . 


\section{Chapter 2}

\section{Mathematical Preliminaries and Notations}

Throughout this paper, the norm $\|$.$\| denotes the usual L^{2}(\Omega)$ norm of scalars, vectors and tensors, induced by the usual $L^{2}$ inner-product, denoted by $(\cdot, \cdot)$. The space in which velocity sought(at time $t)$ is

$$
X=H_{0}^{1}(\Omega)^{d}=\left\{v \in L^{2}(\Omega)^{d}: \nabla v \in L^{2}(\Omega)^{d x d} \text { and } v=0 \text { on } \partial \Omega\right\} \text {. }
$$

with the norm $\|v\|_{X}=\|\nabla v\|$. The space dual to $X$ is equipped with the norm

$$
\|f\|_{-1}=\sup _{v \in X} \frac{(f, v)}{\|\nabla v\|}
$$

The space that velocity (at time $t$ ) belongs to is

$$
Q=L_{0}^{2}(\Omega)=\left\{q \in L^{2}(\Omega): \int_{\Omega} q(x) d x=0\right\}
$$

Introduce the space of weakly divergence-free functions 


$$
X \supset V=\{v \in X:(\nabla \cdot v, q)=0, \forall q \in Q\}
$$

For measurable $v:[0, T] \rightarrow X$, we define

$$
\|v\|_{L^{p}(0, T ; X)}=\left(\int_{0}^{T}\|v\|_{X}^{P} d t\right)^{\frac{1}{p}}, 1 \leq p<\infty
$$

and

$$
\|v\|_{L^{\infty}(0, T ; X)}=e s s \sup _{0 \leq t \leq T}\|v(t)\|_{X} .
$$

Define the trilinear form on $X \times X \times X$

$$
b(u, v, w)=\int_{\Omega} u \cdot \nabla v \cdot w d x
$$

The following lemma is also necessary for the analysis.

Lemma 2.0.1 There exist finite constant $M=M(d)$ and $N=N(d)$ s.t. $M \geq N$ and

$$
M=\sup _{u, v, w \in X} \frac{b(u, v, w)}{\|u|\||| v|\||| w\|}<\infty, N=\sup _{u, v, w \in V} \frac{b(u, v, w)}{\|u\|\|v\|\|w\|}<\infty
$$

The proof can be found in [29]. The corresponding constants $M^{h}$ and $N^{h}$ are defined by replacing $X$ by the finite element space $X^{h} \subset X$ and $V$ by $V^{h} \subset X$. Note that $M \geq \max \left(M^{h}, N, N^{h}\right)$ and that as $h \rightarrow 0, N^{h} \rightarrow N$ and $M^{h} \rightarrow M$ (see [29]).

Throughout the paper, we shall assume that the velocity-pressure finite element spaces $X^{h} \subset X$ and $Q^{h} \subset Q$ are conforming, have typical approximation properties of finite element spaces commonly in use, and satisfy the discrete inf-sup, or $L B B^{h}$, condition

$$
\inf _{q^{h} \in Q^{h}} \sup _{v^{h} \in X^{h}} \frac{\left(q^{h}, \nabla \cdot v^{h}\right)}{\left\|\nabla v^{h}\right\|\left\|q^{h}\right\|} \geq \beta^{h}>0,
$$

where $\beta^{h}$ is bounded away from zero uniformly in $h$. Examples of such spaces can be found in [29]. We shall consider $X^{h} \subset X, Q^{h} \subset Q$ to be spaces of continuous 
piecewise polynomials of degree $m$ and $m-1$, respectively, with $m \geq 2$. The case of $m=1$ is not considered, because the optimal error estimate (of the order $h$ ) is obtained after the first step of the method, and therefore the DCM in this case is reduced to the artificial viscosity approach.

The space of discretely divergence-free functions is defined as follows

$$
V^{h}=\left\{v^{h} \in X^{h}:\left(q^{h}, \nabla \cdot v^{h}\right)=0, \forall q^{h} \in Q^{h}\right\}
$$

In the analysis we use the properties of the following Modified Stokes Projection

Definition 2.0.2 (Modified Stokes Projection) Define the Stokes projection operator $P_{S}:(X, Q) \rightarrow\left(X^{h}, Q^{h}\right), P_{S}(u, p)=(\tilde{u}, \tilde{p})$, satisfying

$$
\begin{aligned}
(h+\nu)\left(\nabla(u-\tilde{u}), \nabla v^{h}\right)-\left(p-\tilde{p}, \nabla \cdot v^{h}\right) & =0 \\
\left(\nabla \cdot(u-\tilde{u}), q^{h}\right) & =0,
\end{aligned}
$$

for any $v^{h} \in V^{h}, q^{h} \in Q^{h}$.

In $\left(V^{h}, Q^{h}\right)$ this formulation reads: given $(u, p) \in(X, Q)$, find $\tilde{u} \in V^{h}$ satisfying

$$
(h+\nu)\left(\nabla(u-\tilde{u}), \nabla v^{h}\right)-\left(p-q^{h}, \nabla \cdot v^{h}\right)=0,
$$

for any $v^{h} \in V^{h}, q^{h} \in Q^{h}$.

Define the explicitly skew-symmetrized trilinear form

$$
b^{*}(u, v, w):=\frac{1}{2}(u \cdot \nabla v, w)-\frac{1}{2}(u \cdot \nabla w, v) .
$$

The following estimate is easy to prove (see, e.g., [29]): there exists a constant $C=C(\Omega)$ such that

$$
\left|b^{*}(u, v, w)\right| \leq C(\Omega)\|\nabla u\|\|\nabla v\|\|\nabla w\| .
$$


The proofs will require the sharper bound on the nonlinearity. This upper bound is improvable in $R^{2}$.

Lemma 2.0.3 (The sharper bound on the nonlinear term) Let $\Omega \subset R^{d}, d=$ 2,3. For all $u, v, w \in X$

$$
\left|b^{*}(u, v, w)\right| \leq C(\Omega) \sqrt{\|u\|\|\nabla u\|}\|\nabla v\|\|\nabla w\|
$$

Proof 2.0.1 See [29].

We will also need the following inequalities: for any $u \in V$

$$
\begin{gathered}
\inf _{v \in V^{h}}\|\nabla(u-v)\| \leq C(\Omega) \inf _{v \in X^{h}}\|\nabla(u-v)\|, \\
\inf _{v \in V^{h}}\|u-v\| \leq C(\Omega) \inf _{v \in X^{h}}\|\nabla(u-v)\|,
\end{gathered}
$$

The proof of (2.0.5) can be found, e.g., in [29], and (2.0.6) follows from the PoincareFriedrich's inequality and (2.0.5).

We will also assume that the inverse inequality holds: there exists a constant $C$ independent of $h$, such that

$$
\|\nabla v\| \leq C h^{-1}\|v\|, \forall v \in X^{h}
$$

Define also the number of time steps $N:=\frac{T}{k}$.

We will use the error decomposition

$$
\begin{array}{r}
e_{\ell}^{i}=u^{i}-u_{\ell}^{h, i}=u^{i}-\tilde{u}^{i}+\tilde{u}^{i}-u_{\ell}^{h, i}=\eta_{\ell}^{i}-\phi_{\ell}^{h, i}, \\
\text { where } \tilde{u}^{i} \in V^{h} \text { is some projection of } u^{i} \text { onto } V^{h}, \\
\text { and } \eta_{\ell}^{i}=u^{i}-\tilde{u}^{i}, \phi_{\ell}^{h, i}=u_{\ell}^{h, i}-\tilde{u}^{i}, \phi_{\ell}^{h, i} \in V^{h}, \forall i, \forall \ell=1,2 .
\end{array}
$$


We conclude the preliminaries by formulating the discrete Gronwall's lemma, see, e.g. $[30]$

Lemma 2.0.4 Let $k, B$, and $a_{\mu}, b_{\mu}, c_{\mu}, \gamma_{\mu}$, for integers $\mu \geq 0$, be nonnegative numbers such that:

$$
a_{n}+k \sum_{\mu=0}^{n} b_{\mu} \leq k \sum_{\mu=0}^{n} \gamma_{\mu} a_{\mu}+k \sum_{\mu=0}^{n} c_{\mu}+B \text { for } n \geq 0 .
$$

Suppose that $k \gamma_{\mu}<1$ for all $\mu$, and set $\sigma_{\mu}=\left(1-k \gamma_{\mu}\right)^{-1}$. Then

$$
a_{n}+k \sum_{\mu=0}^{n} b_{\mu} \leq e^{k \sum_{\mu=0}^{n} \sigma_{\mu} \gamma_{\mu}} \cdot\left[k \sum_{\mu=0}^{n} c_{\mu}+B\right] .
$$

Upon giving the relationship between Reynolds Number and the kinetic viscosity $(\nu)$ below, we will use $\nu$ instead of $R e^{-1}$.

$$
R e=\frac{\rho v L}{\mu}=\frac{v L}{\nu}
$$

where $\mathrm{v}$ is the maximum velocity of the object relative to the fluid, $\mathrm{L}$ is a characteristic linear dimension, $\mu$ is the dynamic viscosity of the fluid and $\rho$ is the density of the fluid. 


\section{Chapter 3}

\section{AV Approximation}

In this section we prove the unconditional stability and error estimate of the discrete artificial viscosity approximation $u_{1}^{h}$ and use this result to prove an error estimate of its time derivative $\frac{d e_{1}}{d t}$. Over $0 \leq t \leq T<\infty$ the approximations $u_{1}^{h}$ is bounded uniformly in $\nu$.

Hence, the formulation (1.0.3) gives $O(h+k)$ accurate, unconditionally stable extension of the artificial viscosity approximation to the time-dependent Navier-Stokes equations.

We start by giving stability and error estimate of the modified Stokes Projection, that we use as the approximation $\tilde{u}^{0}$ to the initial velocity $u_{0}$. 


\subsection{Stokes Projection}

Proposition 3.1.1 (Stability of the Stokes projection) Let u, us satisfy (2.0.3). The following bound holds

$$
\begin{gathered}
(h+\nu)\|\nabla \tilde{u}\|^{2} \leq 2(h+\nu)\|\nabla u\|^{2} \\
+2 d(h+\nu)^{-1} \inf _{q^{h} \in Q^{h}}\left\|p-q^{h}\right\|^{2},
\end{gathered}
$$

where $d$ is the dimension, $d=2,3$.

Proposition 3.1.2 (Error estimate for Stokes Projection). Suppose the discrete infsup condition (2.0.1) holds. Then the error in Stokes Projection satisfies

$$
\begin{aligned}
(h+\nu)\|\nabla(u-\tilde{u})\|^{2} \leq C & {\left[(h+\nu) \inf _{v^{h} \in V^{h}}\left\|\nabla\left(u-v^{h}\right)\right\|^{2}\right.} \\
& \left.+(h+\nu)^{-1} \inf _{q^{h} \in Q^{h}}\left\|p-q^{h}\right\|^{2}\right],
\end{aligned}
$$

where $C$ is a constant independent of $h$ and $R e$.

Proof 3.1.1 Proofs can be found in [15]

\subsection{Stability of the AV approximation}

Lemma 3.2.1 Let $u_{1}^{h}$ satisfy the equation (1.0.3). Let $f \in L^{2}\left(0, T ; H^{-1}(\Omega)\right)$. Then for $n=0, \ldots, N-1$

$$
\begin{gathered}
\left\|u_{1}^{h, n+1}\right\|^{2}+k \sum_{i=1}^{n+1}(h+\nu)\left\|\nabla u_{1}^{h, i}\right\|^{2} \leq\left\|u_{0}^{s}\right\|^{2} \\
+\frac{1}{h+\nu} k \sum_{i=1}^{n+1}\left\|f\left(t_{i}\right)\right\|_{-1}^{2} .
\end{gathered}
$$


Also, if $f \in L^{2}\left(0, T ; L^{2}(\Omega)\right)$ and the time constraint $T$ is finite, then there exists a constant $C=C(T)$ such that

$$
\begin{array}{r}
\left\|u_{1}^{h, n+1}\right\|^{2}+k \sum_{i=1}^{n+1}(h+\nu)\left\|\nabla u_{1}^{h, i}\right\|^{2} \\
\leq C\left(\left\|u_{0}^{s}\right\|^{2}+k \sum_{i=1}^{n+1}\left\|f\left(t_{i}\right)\right\|^{2}\right) .
\end{array}
$$

Proof 3.2.1 Can be found in [15].

\subsection{Error Estimates of AV Approximation}

\section{Definition 3.3.1 Let}

$$
\begin{aligned}
C_{u} & :=\|u(x, t)\|_{L^{\infty}\left(0, T ; L^{\infty}(\Omega)\right)}, \\
C_{\nabla u} & :=\|\nabla u(x, t)\|_{L^{\infty}\left(0, T ; L^{\infty}(\Omega)\right)}, \\
\tilde{C}_{u} & :=\|u(x, t)\|_{L^{\infty}\left(0, T ; L^{2}(\Omega)\right)}, \\
\tilde{C}_{\nabla u} & :=\|\nabla u(x, t)\|_{L^{\infty}\left(0, T ; L^{2}(\Omega)\right)},
\end{aligned}
$$

and introduce $\tilde{C}$, satisfying

$$
\inf _{v \in V^{h}}\|\nabla(u-v)\| \leq C_{1} \inf _{v \in X^{h}}\|\nabla(u-v)\| \leq C_{2} h^{m}\|u\|_{H^{m+1}} \leq \tilde{C} h^{m}
$$

Also, using the constant $C(\Omega)$ from Lemma 2.3, we define $\bar{C}:=1728 C^{4}(\Omega)$.

Theorem 3.3.2 Let $f \in L^{2}\left(0, T ; H^{-1}\right)$, let $u_{1}^{h}, u_{2}^{h}$ satisfy (1.0.3) and (1.0.4), respectively,

$$
\begin{gathered}
k \leq \frac{h+\nu}{4 C_{u}^{2}+2(h+\nu) C_{\nabla u}+2 \bar{C} \tilde{C}^{4}(h+\nu)^{-2} h^{4 m}}, \\
u \in L^{2}\left(0, T ; H^{m+1}(\Omega)\right) \cap L^{\infty}\left(0, T ; L^{\infty}(\Omega)\right), \nabla u \in L^{\infty}\left(0, T, L^{\infty}(\Omega)\right), \\
u_{t} \in L^{2}\left(0, T ; H^{m+1}(\Omega)\right), u_{t t} \in L^{2}\left(0, T ; L^{2}(\Omega)\right), p \in L^{2}\left(0, T ; H^{m}(\Omega)\right) .
\end{gathered}
$$


Then there exist a constant $C=C(\Omega, T, u, p, f, h+\nu)$, such that

$$
\max _{1 \leq i \leq N}\left\|u\left(t_{i}\right)-u_{1}^{h, i}\right\|+\left(k \sum_{i=1}^{n+1}(h+\nu)\left\|\nabla\left(u\left(t_{i}\right)-u_{1}^{h, i}\right)\right\|^{2}\right)^{1 / 2} \leq C\left(h^{m}+h+k\right)
$$

Proof 3.3.1 Can be found [15].

We will need the following lemma in the proof of Theorem (3.3.4).

Lemma 3.3.3 Let $f \in L^{2}\left(0, T ; H^{-1}(\Omega)\right)$. Suppose $\phi^{0}$ and $\phi^{1}$ to be the Stokes projections of the initial velocity and velocity at the first time level, respectively. Let $m \geq 2$ and

$$
k<\frac{4(h+\nu)}{13\left(4(h+\nu) C_{\nabla u}+3 C_{u}^{2}\right)} .
$$

Then there exist a constant $C=C(\Omega, T, u, p, f, h+\nu)$, such that

$$
\left\|\frac{\phi^{1}-\phi^{0}}{k}\right\|^{2}+\frac{13}{2}(h+\nu) k\left\|\nabla \frac{\phi^{1}-\phi^{0}}{k}\right\|^{2} \leq C\left(k h^{2 m}+h^{2}+k^{2}+k^{2} h^{2 m-3}\right)
$$

Proof 3.3.2 From the Stokes Projection(2.0.2) and error decomposition(2.0.8), we have

$$
(h+\nu)\left(\nabla \phi^{0}, \nabla v\right)-(h+\nu)\left(\nabla \eta^{0}, \nabla v\right)-\left(p^{0}-q, \nabla . v\right)=0
$$

On the other hand the solution at the first time level satisfies the following 


$$
\begin{array}{r}
\left\|\frac{\phi^{1}-\phi^{0}}{k}\right\|^{2}+(h+\nu)\left(\nabla \phi^{1}, \nabla \frac{\phi^{1}-\phi^{0}}{k}\right)+b^{*}\left(u^{1}, u^{1}, \frac{\phi^{1}-\phi^{0}}{k}\right) \\
-b^{*}\left(u_{1}^{1}, u_{1}^{1}, \frac{\phi^{1}-\phi^{0}}{k}\right)+\left(p^{1}, \nabla \cdot \frac{\phi^{1}-\phi^{0}}{k}\right) \\
=h\left(\nabla u^{1}, \nabla \frac{\phi^{1}-\phi^{0}}{k}\right)+k\left(\rho^{1}, \frac{\phi^{1}-\phi^{0}}{k}\right) \\
+\left(\frac{\eta^{1}-\eta^{0}}{k}, \frac{\phi^{1}-\phi^{0}}{k}\right)+(h+\nu)\left(\nabla \eta^{1}, \frac{\phi^{1}-\phi^{0}}{k}\right),
\end{array}
$$

where $k \rho^{1}=\frac{u^{1}-u^{0}}{k}-u_{t}^{1}=k u_{t t}^{\theta}$, for some $\theta \in(0, k)$.

Subtracting equation 3.3.3 from equation 3.3.4 for $v=\frac{\phi^{1}-\phi^{0}}{k}$, we have

$$
\begin{array}{r}
\left\|\frac{\phi^{1}-\phi^{0}}{k}\right\|^{2}+k(h+\nu)\left\|\nabla \frac{\phi^{1}-\phi^{0}}{k}\right\|^{2} \\
+b^{*}\left(u^{1}, u^{1}, \frac{\phi^{1}-\phi^{0}}{k}\right)-b^{*}\left(u_{1}^{1}, u_{1}^{1}, \frac{\phi^{1}-\phi^{0}}{k}\right) \\
-k\left(\frac{p^{1}-p^{0}}{k}-q, \nabla \cdot \frac{\phi^{1}-\phi^{0}}{k}\right) \\
=h\left(\nabla u^{1}, \nabla \frac{\phi^{1}-\phi^{0}}{k}\right)+\left(\rho^{1}, \frac{\phi^{1}-\phi^{0}}{k}\right)+\left(\frac{\eta^{1}-\eta^{0}}{k}, \frac{\phi^{1}-\phi^{0}}{k}\right) \\
+k(h+\nu)\left(\nabla \frac{\eta^{1}-\eta^{0}}{k}, \nabla \frac{\phi^{1}-\phi^{0}}{k}\right)
\end{array}
$$

Adding and subtracting $b^{*}\left(u_{1}^{1}, u^{1}, \frac{\phi^{1}-\phi^{0}}{k}\right)$ to the nonlinear terms in equation (3.3.5) together with error decomposition (2.0.8) gives

$$
\begin{aligned}
b^{*}\left(u^{1}, u^{1}, \frac{\phi^{1}-\phi^{0}}{k}\right)- & b^{*}\left(u_{1}^{1}, u_{1}^{1}, \frac{\phi^{1}-\phi^{0}}{k}\right) \\
= & b^{*}\left(e_{1}^{1}, u^{1}, \frac{\phi^{1}-\phi^{0}}{k}\right)+b^{*}\left(u_{1}^{1}, e_{1}^{1}, \frac{\phi^{1}-\phi^{0}}{k}\right) \\
= & b^{*}\left(\phi^{1}, u^{1}, \frac{\phi^{1}-\phi^{0}}{k}\right)-b^{*}\left(\eta^{1}, u^{1}, \frac{\phi^{1}-\phi^{0}}{k}\right) \\
& +b^{*}\left(u_{1}^{1}, \phi^{1}, \frac{\phi^{1}-\phi^{0}}{k}\right)-b^{*}\left(u_{1}^{1}, \eta^{1}, \frac{\phi^{1}-\phi^{0}}{k}\right)
\end{aligned}
$$


Adding and subtracting $\phi^{0}$ to the first component of the first nonlinear term in the equation (3.3.6) gives

$$
b^{*}\left(\phi^{1}, u^{1}, \frac{\phi^{1}-\phi^{0}}{k}\right)=k b^{*}\left(\frac{\phi^{1}-\phi^{0}}{k}, u^{1}, \frac{\phi^{1}-\phi^{0}}{k}\right)+b^{*}\left(\phi^{0}, u^{1}, \frac{\phi^{1}-\phi^{0}}{k}\right)
$$

In the first nonlinear term of (3.3.7), applying Cauchy-Schwarz and Young's inequalities together with the regularity assumption of $u$ and bound 2.0.4 gives

$$
\begin{array}{r}
k\left|b^{*}\left(\frac{\phi^{1}-\phi^{0}}{k}, u^{1}, \frac{\phi^{1}-\phi^{0}}{k}\right)\right| \leq k C_{\nabla u}\left\|\frac{\phi^{1}-\phi^{0}}{k}\right\|^{2} \\
+k \mu^{*}(h+\nu)\left\|\nabla \frac{\phi^{1}-\phi^{0}}{k}\right\|^{2}+k \frac{C_{u}^{2}}{16(h+\nu) \mu^{*}}\left\|\frac{\phi^{1}-\phi^{0}}{k}\right\|^{2}
\end{array}
$$

In the second nonlinear term of (3.3.7), applying Cauchy Schwarz and Young's inequalities together with bound 2.0.4 and inverse inequality (2.0.7) gives

$$
\left|b^{*}\left(\phi^{0}, u^{1}, \frac{\phi^{1}-\phi^{0}}{k}\right)\right| \leq \mu|| \frac{\phi^{1}-\phi^{0}}{k}\left\|^{2}+\frac{C h^{-2}}{4 \mu}\right\| \nabla \phi^{0} \|^{2}
$$

In the second nonlinear term of (3.3.6), applying Cauchy Schwarz and Young's inequalities together with bound 2.0.4 and inverse inequality (2.0.7) gives

$$
\left|b^{*}\left(\eta^{1}, u^{1}, \frac{\phi^{1}-\phi^{0}}{k}\right)\right| \leq \mu|| \frac{\phi^{1}-\phi^{0}}{k}\left\|^{2}+\frac{C h^{-2}}{4 \mu}\right\| \nabla \eta^{1} \|^{2}
$$

For the third nonlinear term of equation (3.3.6), applying error decomposition (2.0.8) gives

$$
\begin{aligned}
\left|b^{*}\left(u_{1}^{1}, \phi^{1}, \frac{\phi^{1}-\phi^{0}}{k}\right)\right| \leq\left|b^{*}\left(u^{1}, \phi^{1}, \frac{\phi^{1}-\phi^{0}}{k}\right)\right| & +\left|b^{*}\left(\phi^{1}, \phi^{1}, \frac{\phi^{1}-\phi^{0}}{k}\right)\right| \\
& +\left|b^{*}\left(\eta^{1}, \phi^{1}, \frac{\phi^{1}-\phi^{0}}{k}\right)\right|
\end{aligned}
$$


Since nonlinear form is skew-symmetric in the second and third entry, we can replace terms like the first nonlinear term in the inequality (3.3.11) with terms like $\left|b^{*}\left(u^{1}, \phi^{0}, \frac{\phi^{1}-\phi^{0}}{k}\right)\right|$. Applying Cauchy-Schwarz and Young's inequalities together with the regularity assumption of $u$ and inverse inequality gives

$$
\left|b^{*}\left(u^{1}, \phi^{0}, \frac{\phi^{1}-\phi^{0}}{k}\right)\right| \leq 2 \mu\left\|\frac{\phi^{1}-\phi^{0}}{k}\right\|^{2}+\frac{C_{u}^{2}}{4 \mu}\left(\left\|\nabla \phi^{0}\right\|^{2}+h^{-2}\left\|\phi^{0}\right\|^{2}\right)
$$

Applying Young's inequality together with the sharper bound (2.0.3) and inverse inequality (2.0.7) in the second nonlinear term of 3.3 .11 gives

$$
\left|b^{*}\left(\phi^{1}, \phi^{1}, \frac{\phi^{1}-\phi^{0}}{k}\right)\right|=\left|b^{*}\left(\phi^{1}, \phi^{0}, \frac{\phi^{1}-\phi^{0}}{k}\right)\right| \leq \mu|| \frac{\phi^{1}-\phi^{0}}{k}\left\|^{2}+\frac{C h^{-3}}{4 \mu}\right\| \phi^{1}\left\|^{2}\right\| \nabla \phi^{0} \|^{2}
$$

For the last nonlinear term in the inequality (3.3.11), we can apply 2.0.4 and inverse inequality followed by Young's inequality to have

$$
\begin{gathered}
\left|b^{*}\left(\eta^{1}, \phi^{1}, \frac{\phi^{1}-\phi^{0}}{k}\right)\right|=\left|b^{*}\left(\eta^{1}, \phi^{0}, \frac{\phi^{1}-\phi^{0}}{k}\right)\right| \\
\leq \mu\left\|\frac{\phi^{1}-\phi^{0}}{k}\right\|^{2}+\left.\frac{C h^{-2}}{4 \mu}\left\|\nabla \eta^{1}\right\|\right|^{2}\left\|\nabla \phi^{0}\right\|^{2}
\end{gathered}
$$

For the forth nonlinear term of equation (3.3.6), applying error decomposition gives

$$
\begin{aligned}
\left|b^{*}\left(u_{1}^{1}, \eta^{1}, \frac{\phi^{1}-\phi^{0}}{k}\right)\right| \leq\left|b^{*}\left(u^{1}, \eta^{1}, \frac{\phi^{1}-\phi^{0}}{k}\right)\right| & +\left|b^{*}\left(\phi^{1}, \eta^{1}, \frac{\phi^{1}-\phi^{0}}{k}\right)\right| \\
& +\left|b^{*}\left(\eta^{1}, \eta^{1}, \frac{\phi^{1}-\phi^{0}}{k}\right)\right|
\end{aligned}
$$

For all the nonlinear terms in the inequality (3.3.15), we can apply bound 2.0.4 and inverse inequality followed by Young's inequality to have 


$$
\begin{gathered}
\left|b^{*}\left(u^{1}, \eta^{1}, \frac{\phi^{1}-\phi^{0}}{k}\right)\right| \leq \mu|| \frac{\phi^{1}-\phi^{0}}{k}\left\|^{2}+\frac{C h^{-2}}{4 \mu}\right\| \nabla \eta^{1} \|^{2} \\
\left|b^{*}\left(\phi^{1}, \eta^{1}, \frac{\phi^{1}-\phi^{0}}{k}\right)\right| \leq \mu|| \frac{\phi^{1}-\phi^{0}}{k}\left\|^{2}+C h^{-4}\right\| \nabla \eta^{1}\left\|^{2}\right\| \phi^{1} \|^{2} \\
\left.\left|b^{*}\left(\eta^{1}, \eta^{1}, \frac{\phi^{1}-\phi^{0}}{k}\right)\right| \leq \mu|| \frac{\phi^{1}-\phi^{0}}{k}\right)\left\|^{2}+C h^{-2}\right\| \nabla \eta^{1} \|^{4}
\end{gathered}
$$

Apply Cauchy-Schwarz and Young's inequalities to (3.3.5).

Since $\left\|\nabla \cdot \frac{\phi^{1}-\phi^{0}}{k}\right\| \leq d\left\|\nabla \frac{\phi^{1}-\phi^{0}}{k}\right\|$,

$$
\begin{array}{r}
\left(1-12 \mu-\left(\frac{C_{\nabla u}}{2}+\frac{C_{u}^{2}}{16(h+\nu) \mu^{*}}\right) k\right)\left\|\frac{\phi^{1}-\phi^{0}}{k}\right\|^{2} \\
+\left(1-3 \mu^{*}\right)(h+\nu) k\left\|\nabla \frac{\phi^{1}-\phi^{0}}{k}\right\|^{2} \\
\leq \frac{d k}{4 \mu^{*}(h+\nu)} \inf _{q \in Q^{h}}\left\|\frac{p^{1}-p^{0}}{k}-q\right\|^{2}+\frac{h^{2}}{4 \mu}\left\|\Delta u^{1}\right\|^{2}+\frac{k^{2}}{4 \mu}\left\|\rho^{1}\right\|^{2}+\frac{1}{4 \mu}\left\|\frac{\eta^{1}-\eta^{0}}{k}\right\|^{2} \\
+\frac{k(h+\nu)}{4 \mu^{*}}\left\|\nabla \frac{\eta^{1}-\eta^{0}}{k}\right\|^{2}+\frac{C h^{-2}}{4 \mu}\left\|\nabla \phi^{0}\right\|^{2}+\frac{C_{u}^{2}}{4 \mu}\left\|\nabla \phi^{0}\right\|^{2}+\frac{C_{u}^{2} h^{-2}}{4 \mu}\left\|\phi^{0}\right\|^{2} \\
+\frac{C h^{-3}}{4 \mu}\left\|\phi^{1}\right\|^{2}\left\|\nabla \phi^{0}\right\|^{2}+\frac{C h^{-2}}{4 \mu}\left\|\nabla \eta^{1}\right\|^{2}\left\|\nabla \phi^{0}\right\|^{2} \\
+\frac{C h^{-2}}{2 \mu}\left\|\nabla \eta^{1}\right\|^{2}+C h^{-4}\left\|\phi^{1}\right\|^{2}\left\|\nabla \eta^{1}\right\|^{2}+C h^{-2}\left\|\nabla \eta^{1}\right\|^{4}
\end{array}
$$

Use the approximation properties of $X^{h}, Q^{h}$. Since the mesh nodes do not depend upon the time level, it follows from (2.0.5), (2.0.6) that 


$$
\begin{array}{r}
\inf _{q \in Q}\left\|\frac{p^{1}+p^{0}}{k}-q\right\|^{2} \leq C h^{2 m}, \\
\left\|\frac{\eta_{2}^{1}-\eta_{2}^{0}}{k}\right\|^{2} \leq C h^{2 m+2}, \\
\left\|\eta_{2}^{1}\right\|^{2} \leq C h^{2 m+2} .
\end{array}
$$

Taking $\mu=1 / 13$ and $\mu^{*}=1 / 6$ and using bounds (3.3.20) for each term, it follows from the regularity assumption of $u$ that

$$
\begin{array}{r}
\left(\frac{1}{13}-\left(\frac{C_{\nabla u}}{2}+\frac{3 C_{u}^{2}}{8(h+\nu)}\right) k\right)\left\|\frac{\phi^{1}-\phi^{0}}{k}\right\|^{2}+\frac{1}{2}(h+\nu) k\left\|\nabla \frac{\phi^{1}-\phi^{0}}{k}\right\|^{2} \\
\leq C\left(h^{2 m-2}+h^{2}+k^{2}+k^{2} h^{2 m-3}\right)
\end{array}
$$

The last inequality implies the lemma statement.

Theorem 3.3.4 Let the assumptions of Lemma (3.3.3) and Theorem (3.3.2) be satisfied.

Let $k \leq \min \left\{\frac{h+\nu}{2 C C_{\nabla u}(h+\nu)+2 C C_{u}^{2}}, C(h+\nu)^{\frac{5}{3}}, C(h+\nu)^{3}\right\}$

Then

$$
\left\|\frac{e_{1}^{n+1}-e_{1}^{n}}{k}\right\|^{2}+k \sum_{i=0}^{n}(h+\nu)\left\|\nabla \frac{e_{1}^{i+1}-e_{1}^{i}}{k}\right\|^{2} \leq C\left[h^{2 m}+h^{2}+k^{2}\right]
$$

Proof 3.3.3 Start with the proof of the bound for $\left\|\frac{\phi^{n+1}-\phi^{n}}{k}\right\|$.

From the inequality (5.14) in [15], we have 


$$
\begin{array}{r}
\left\|s^{h, n+1}\right\|^{2}+k(h+\nu) \sum_{i=1}^{n}\left\|\nabla s^{h, i+1}\right\|^{2} \\
\leq\left\|s^{h, 1}\right\|^{2}+C\left[h^{2 m}+h^{2}+k^{2}\right] \\
+C k \sum_{i=1}^{n}\left(C_{\nabla u}+\frac{C_{u}^{2}}{h+\nu}+\frac{1}{(h+\nu)^{3}}\left\|\nabla e_{1}^{i}\right\|^{4}\right)\left\|s^{h, i+1}\right\|^{2}, \\
\text { where } s^{h, n+1}=\frac{\phi^{n+1}-\phi^{n}}{k}
\end{array}
$$

In order to apply Gronwall's Lemma 2.0.4 in the inequality 3.3.22, we have to verify that

$$
C k\left(C_{\nabla u}+\frac{C_{u}^{2}}{h+\nu}+\frac{1}{(h+\nu)^{3}}\left\|\nabla e_{1}^{i}\right\|^{4}\right)<1 .
$$

To this end, we can first assume

$$
C k\left(C_{\nabla u}+\frac{C_{u}^{2}}{h+\nu}\right)<\frac{1}{2} \text { and } \frac{C k}{(h+\nu)^{3}}\left\|\nabla e_{1}^{i}\right\|^{4}<\frac{1}{2} .
$$

Due to the first inequality, we have a bound on $k$ in the form

$$
k<\frac{h+\nu}{C C_{\nabla u}(h+\nu)+C C_{u}^{2}} .
$$

For the second inequality we investigate case by case.

For $k \leq h$, it follows from the inverse inequality and theorem (3.3.2) that

$$
\begin{aligned}
\frac{C k}{(h+\nu)^{3}}\left\|\nabla e_{1}^{i}\right\|^{4} \leq \frac{C k h^{-4}}{(h+\nu)^{3}}\left\|e_{1}^{i}\right\|^{4} \leq & \frac{C k}{(h+\nu)^{3}}\left(1+\frac{k}{h}\right)^{4} \\
& \leq \frac{C k}{(h+\nu)^{3}}<\frac{1}{2} .
\end{aligned}
$$

Thus, we have a bound on $k$ in the form $k<C(h+\nu)^{3}$. 
For $h \leq k$, it follows from the theorem (3.3.2) that

$$
\frac{C k}{(h+\nu)^{3}}\left\|\nabla e_{1}^{i}\right\|^{4} \leq \frac{C k^{-1}}{(h+\nu)^{5}}\left(h^{4}+k^{4}\right) \leq \frac{2 C k^{3}}{(h+\nu)^{5}}<\frac{1}{2} .
$$

It follows from the above calculations and theorem statement that

$$
\left(C_{\nabla u}+\frac{C_{u}^{2}}{h+\nu}+\frac{1}{(h+\nu)^{3}}\left\|\nabla e_{1}^{i}\right\|^{4}\right) k<1 .
$$

Now, we can apply discrete Gronwall's Lemma in the inequality (3.3.22) to have following bound

$$
\left\|\frac{\phi^{n+1}-\phi^{n}}{k}\right\|^{2}+(h+\nu) k \sum_{i=1}^{n}\left\|\nabla \frac{\phi^{i+1}-\phi^{i}}{k}\right\|^{2} \leq C\left[h^{2 m}+h^{2}+k^{2}\right]
$$

Using the triangle inequality in the error decomposition (2.0.8), we obtain

$$
\left\|\frac{e_{1}^{n+1}-e_{1}^{n}}{k}\right\|^{2}+k \sum_{i=0}^{n}(h+\nu)\left\|\nabla \frac{e_{1}^{n+1}-e_{1}^{n}}{k}\right\|^{2} \leq C\left[h^{2 m}+h^{2}+k^{2}\right]
$$

This result proves the theorem. 


\section{Chapter 4}

\section{Correction Step Approximation}

In this section we prove the unconditional stability and error estimate of the correction step approximation $u_{2}^{h}$. Over $0 \leq t \leq T<\infty$ the approximations $u_{2}^{h}$ is bounded uniformly in $R e$.

Hence, the formulation (1.0.4) gives $O\left(h^{2}+k^{2}\right)$ accurate, unconditionally stable extension of correction step approximation to the time-dependent Navier-Stokes equations.

We start by proving stability of correction step approximation.

\subsection{Stability of the CS Approximation}

Theorem 4.1.1 Let $f \in L^{2}\left(0, T ; H^{-1}(\Omega)\right)$, let $u_{1}^{h}, u_{2}^{h}$ satisfy (1.0.3) and (1.0.4), respectively. Then for $n=0, \ldots, N-1$, 


$$
\begin{array}{r}
\left\|u_{2}^{h, n+1}\right\|^{2}+5 h^{2}(h+\nu)^{-2}\left\|u_{1}^{h, n+1}\right\|^{2}+k \sum_{i=1}^{n+1}(h+\nu)\left\|\nabla u_{2}^{h, i}\right\|^{2} \\
\leq C\left[\left\|u_{0}^{s}\right\|^{2}+(h+\nu)^{-1} k \sum_{i=1}^{n+1}\left\|f\left(t_{i}\right)\right\|_{-1}^{2}\right] .
\end{array}
$$

Proof 4.1.1 Take $v^{h}=u_{2}^{h, n+1} \in V^{h}$ in the equation (1.0.4). This gives with CauchySchwarz and Young's inequality that

$$
\begin{array}{r}
\frac{1}{2 k}\left(\left\|u_{2}^{h, n+1}\right\|^{2}-\left\|u_{2}^{h, n}\right\|^{2}\right)+(h+\nu)\left\|\nabla u_{2}^{h, n+1}\right\|^{2} \\
\leq\left(\frac{f\left(t_{n+1}\right)+f\left(t_{n}\right)}{2}, u_{2}^{h, n+1}\right)+\frac{\nu}{2} k\left(\nabla\left(\frac{u_{1}^{n+1}-u_{1}^{n+1}}{k}, \nabla u_{2}^{h, n+1}\right)\right. \\
+\frac{1}{2} b^{*}\left(u_{1}^{h, n+1}, u_{1}^{h, n+1}, u_{2}^{h, n+1}\right)-\frac{1}{2} b^{*}\left(u_{1}^{h, n}, u_{1}^{h, n}, u_{2}^{h, n+1}\right)+h\left(\nabla u_{1}^{h, n}, \nabla u_{2}^{h, n}\right)
\end{array}
$$

It follows from Cauchy-Schwarz, Young's and triangle inequalities with the error estimate $e_{1}^{i}=u\left(t_{i}\right)-u_{1}^{i}$ that

$$
\begin{array}{r}
\frac{\nu}{2} k\left(\nabla\left(\frac{u_{1}^{n+1}-u_{1}^{n}}{k}, \nabla u_{2}^{h, n+1}\right) \leq \mu(h+\nu)\left\|\nabla u_{2}^{h, n+1}\right\|^{2}\right. \\
+\frac{\nu^{2} k^{2}}{8 \mu(h+\nu)}\left\|\nabla\left(\frac{u^{n+1}-u^{n}}{k}\right)\right\|^{2}+\frac{\nu^{2} k}{8 \mu(h+\nu)} k\left\|\nabla\left(\frac{e_{1}^{n+1}-e_{1}^{n}}{k}\right)\right\|^{2} .
\end{array}
$$

Adding and subtracting $\frac{1}{2} b^{*}\left(u_{1}^{h, n+1}, u_{1}^{h, n}, u_{2}^{h, n+1}\right)$ to the nonlinear terms and applying the bound (2.0.4) followed by Cauchy-Schwarz, Young's and triangle inequalities with regularity assumption of $u$, we have 


$$
\begin{array}{r}
\frac{1}{2} b^{*}\left(u_{1}^{h, n+1}, u_{1}^{h, n+1}, u_{2}^{h, n+1}\right)-\frac{1}{2} b^{*}\left(u_{1}^{h, n}, u_{1}^{h, n}, u_{2}^{h, n+1}\right) \\
\leq \frac{1}{2}\left[k b^{*}\left(u_{1}^{h, n+1}, \frac{u_{1}^{h, n+1}-u_{1}^{h, n}}{k}, u_{2}^{h, n+1}\right)+k b^{*}\left(\frac{u_{1}^{h, n+1}-u_{1}^{h, n}}{k}, u_{1}^{h, n}, u_{2}^{h, n+1}\right)\right] \\
\leq 2 \mu(h+\nu)\left\|\nabla u_{2}^{h, n+1}\right\|^{2} \\
+\frac{1}{16 \mu(h+\nu)^{3}}(h+\nu) k\left\|\nabla\left(\frac{u_{1}^{h, n+1}-u_{1}^{h, n}}{k}\right)\right\|^{2}\left[(h+\nu) k\left\|\nabla u_{1}^{h, n+1}\right\|^{2}\right. \\
\left.+(h+\nu) k\left\|\nabla u_{1}^{h, n}\right\|^{2}\right] \\
+\frac{1}{8 \mu(h+\nu)^{3}}(h+\nu) k\left\|\nabla\left(\frac{e_{1}^{h, n+1}-e_{1}^{h, n}}{k}\right)\right\|^{2}\left[(h+\nu) k\left\|\nabla u_{1}^{h, n+1}\right\|^{2}\right. \\
+\frac{1}{8 \mu(h+\nu)^{2}} k C_{\nabla u_{t}}^{2}\left[(h+\nu) k\left\|\nabla u_{1}^{h, n+1}\right\|^{2}+(h+\nu) k\left\|\nabla u_{1}^{h, n}\right\|^{2}\right], \\
\quad w h e r e u_{\nabla}^{h, n+1} \|^{2}
\end{array}
$$

Cauchy-Schwarz and Young's inequalities with $\mu=1 / 10$ give

$$
\begin{array}{r}
\frac{1}{2 k}\left(\left\|u_{2}^{h, n+1}\right\|^{2}-\left\|u_{2}^{h, n}\right\|^{2}\right)+\frac{1}{2}(h+\nu)\left\|\nabla u_{2}^{h, n+1}\right\|^{2} \\
\leq \frac{5}{2(h+\nu)}\left\|\frac{f\left(t_{n+1}\right)-f\left(t_{n}\right)}{2}\right\|_{-1}^{2} \\
+\frac{5 \nu^{2} k^{2}}{4(h+\nu)} C_{\nabla u_{t}}^{2}+\frac{5 \nu^{2} k}{4(h+\nu)^{2}} k(h+\nu)\left\|\nabla\left(\frac{e_{1}^{n+1}-e_{1}^{n}}{k}\right)\right\|^{2} \\
+\frac{5 h^{2}}{2(h+\nu)^{2}}(h+\nu)\left\|\nabla u_{1}^{h, n+1}\right\|^{2} \\
+\frac{5}{4(h+\nu)^{3}}(h+\nu) k\left\|\nabla\left(\frac{e_{1}^{h, n+1}-e_{1}^{h, n}}{k}\right)\right\|^{2}\left[(h+\nu) k\left\|\nabla u_{1}^{h, n+1}\right\|^{2}\right. \\
+\frac{5}{4(h+\nu)^{2}} k C_{\nabla u_{t}}^{2}\left[(h+\nu) k\left\|\nabla u_{1}^{h, n+1}\right\|^{2}+(h+\nu) k\left\|\nabla u_{1}^{h, n}\right\|^{2}\right]
\end{array}
$$


Multiplying inequality by $2 k$ and summing over all time levels followed by Lemma (3.2.1) and Theorem (3.3.4) give

$$
\begin{array}{r}
\left\|u_{2}^{h, n+1}\right\|^{2}+\sum_{i=1}^{n+1}(h+\nu)\left\|\nabla u_{2}^{h, i}\right\|^{2} \\
\leq\left\|u_{0}^{s}\right\|^{2}+\frac{5}{(h+\nu)} k \sum_{i=1}^{n+1}\left\|\frac{f\left(t_{i}\right)-f\left(t_{i-1}\right)}{2}\right\|_{-1}^{2} \\
+\frac{5 \nu^{2} k^{3}}{2(h+\nu)} C_{\nabla u_{t}}^{2}+\frac{5 \nu^{2} k^{2}}{2(h+\nu)^{2}} C\left(h^{2 m}+h^{2}+k^{2}\right) \\
+\frac{5 h^{2}}{(h+\nu)^{2}}\left(\left\|u_{0}^{s}\right\|^{2}-\left\|u_{1}^{h, n+1}\right\|^{2}+\frac{1}{h+\nu} k \sum_{i=1}^{n+1}\left\|f\left(t_{i}\right)\right\|_{-1}^{2}\right) \\
+\frac{5}{2(h+\nu)^{2}}\left(\frac{\left(h^{2 m}+h^{2}+k^{2}\right)}{(h+\nu)}+k C_{\nabla u_{t}}^{2}\right)\left[2\left\|u_{0}^{s}\right\|^{2}\right. \\
\left.+\frac{1}{h+\nu} k \sum_{i=1}^{n+1}\left\|f\left(t_{i}\right)\right\|_{-1}^{2}+\frac{1}{h+\nu} k \sum_{i=1}^{n+1}\left\|f\left(t_{i}\right)\right\|_{-1}^{2}\right]
\end{array}
$$

After some algebraic manipulation, we have the following inequality

$$
\begin{array}{r}
\left\|u_{2}^{h, n+1}\right\|^{2}+\frac{5 h^{2}}{(h+\nu)^{2}}\left\|u_{1}^{h, n+1}\right\|^{2}+\sum_{i=1}^{n+1}(h+\nu)\left\|\nabla u_{2}^{h, i}\right\|^{2} \\
\leq\left\|u_{0}^{s}\right\|^{2}+\frac{5}{(h+\nu)} k \sum_{i=1}^{n+1}\left\|\frac{f\left(t_{i}\right)-f\left(t_{i-1}\right)}{2}\right\|_{-1}^{2} \\
+\frac{5 \nu^{2} k^{3}}{2(h+\nu)} C_{\nabla u_{t}}^{2}+\frac{5 \nu^{2} k^{2}}{2(h+\nu)^{2}} C\left(h^{2 m}+h^{2}+k^{2}\right) \\
+C\left(\left\|u_{0}^{s}\right\|^{2}+\frac{1}{h+\nu} k \sum_{i=1}^{n+1}\left\|f\left(t_{i}\right)\right\|_{-1}^{2}\right)
\end{array}
$$

The last inequality implies the theorem statement.

The result of Theorem (4.1.1), combined with the result Proposition (3.1.1), proves the unconditional stability of both $u_{1}^{h, i}$ and $u_{2}^{h, i}$ for any $i \geq 0$.

Next we will prove the error estimate of correction step approximation. 


\subsection{Error Estimate of CS Approximation}

Theorem 4.2.1 Let the assumptions of Theorem (3.3.4) be satisfied. Let

$$
k<\frac{h+\nu}{(h+\nu) C_{\nabla u}+2 C_{u}^{2}+(h+\nu) C h^{m-1}+2 C h^{2 m}} .
$$

Then there exists a constant $C=C(\Omega, T, u, p, f, h+\nu)$, such that

$$
\begin{array}{r}
\max _{1 \leq i \leq N}\left\|u\left(t_{i}\right)-u_{2}^{h, i}\right\|+\left(k \sum_{i=0}^{n}(h+\nu)\left\|\nabla\left(u\left(t_{i}\right)-u_{2}^{h, i}\right)\right\|^{2}\right)^{1 / 2} \\
\leq C\left(h^{m}+h^{2}+k^{2}+h k\right) .
\end{array}
$$

Proof 4.2.1 By Taylor expansion around $t=\frac{t_{n+1}+t_{n}}{2}$, we have $\frac{u^{n+1}-u^{n}}{k}-\frac{u_{t}^{n+1}+u_{t}^{n}}{2}=$ $k^{2} \rho^{n+1}$, where $\rho^{n+1}=\frac{u_{t t t}^{n+\frac{1}{2}}}{8}$.

Summing variational formulations of NSE at $t=t_{n}$ and at $t=t_{n+1}$, and then, dividing by 2, we have the following equation.

$$
\begin{array}{r}
\left(\frac{u^{n+1}-u^{n}}{k}, v\right)+\frac{\nu}{2}\left(\nabla\left(\frac{u^{n+1}+u^{n}}{2}\right), \nabla v\right)+\frac{1}{2} b^{*}\left(u^{n+1}, u^{n+1}, v\right) \\
+\frac{1}{2} b^{*}\left(u^{n}, u^{n}, v\right)-\left(\frac{p^{n+1}+p^{n}}{2}, \nabla \cdot v\right) \\
=\left(\frac{f\left(t_{n+1}\right)+f\left(t_{n}\right)}{2}, v\right)-\left(\frac{u_{t}^{n+1}+u_{t}^{n}}{2}, v\right)+\left(\frac{u^{n+1}-u^{n}}{k}, v\right)
\end{array}
$$

Subtracting (1.0.4) from the equation (4.2.1) and using error decomposition (2.0.8), we have 


$$
\begin{array}{r}
\left(\frac{\phi_{2}^{h, n+1}-\phi_{2}^{h, n}}{k}, \phi_{2}^{h, n+1}\right)+(h+\nu)\left(\nabla \phi_{2}^{h, n+1}, \nabla \phi_{2}^{h, n+1}\right) \\
=\frac{\nu}{2} k\left(\nabla\left(\frac{e_{1}^{h, n+1}-e_{1}^{h, n}}{k}\right), \nabla \phi_{2}^{h, n+1}\right)+\left(\frac{p^{h, n+1}+p^{h, n}}{2}-p_{2}^{n+1}, \nabla . \phi_{2}^{h, n+1}\right) \\
-b^{*}\left(u^{n+1}, \phi_{2}^{h, n+1}, \phi_{2}^{h, n+1}\right)+b^{*}\left(u^{n+1}, \eta_{2}^{n+1}, \phi_{2}^{h, n+1}\right) \\
-b^{*}\left(\phi_{2}^{h, n+1}, u_{2}^{h, n+1}, \phi_{2}^{h, n+1}\right)+b^{*}\left(\eta_{2}^{n+1}, u_{2}^{h, n+1}, \phi_{2}^{h, n+1}\right) \\
+\frac{1}{2} k b^{*}\left(\frac{u^{n+1}-u^{n}}{k}, e_{1}^{h, n}, \phi_{2}^{h, n+1}\right)+\frac{1}{2} k b^{*}\left(u^{n+1}, \frac{e_{1}^{h, n+1}-e_{1}^{h, n}}{k}, \phi_{2}^{h, n+1}\right) \\
+\frac{1}{2} k b^{*}\left(e_{1}^{h, n+1}, \frac{u_{1}^{h, n+1}-u_{1}^{h, n}}{k}, \phi_{2}^{h, n+1}\right)+\frac{1}{2} k b^{*}\left(\frac{e_{1}^{h, n+1}-e_{1}^{h, n}}{k}, u_{1}^{h, n}, \phi_{2}^{h, n+1}\right) \\
h\left(\nabla e_{1}^{h, n+1}, \nabla \phi_{2}^{h, n+1}\right)+k^{2}\left(\rho^{n+1}, \phi_{2}^{h, n+1}\right)+\left(\frac{\eta_{2}^{n+1}-\eta_{2}^{n}}{k}, \phi_{2}^{h, n+1}\right) \\
+(h+\nu)\left(\nabla \eta_{2}^{n+1}, \nabla \phi_{2}^{h, n+1}\right)
\end{array}
$$

We bound the nonlinear terms on the right hand side of (4.2.2), starting now with the second, fifth and sixth terms. Use the bound (2.0.4), regularity assumption of $u$ and Young's inequality to obtain

$$
\begin{gathered}
\left|b^{*}\left(u^{n+1}, \eta_{2}^{n+1}, \phi_{2}^{h, n+1}\right)\right| \leq \mu(h+\nu)\left\|\nabla \phi_{2}^{h, n+1}\right\|^{2} \\
+\frac{C_{\nabla u}^{2}}{4 \mu(h+\nu)}\left\|\nabla \eta_{2}^{n+1}\right\|^{2} \\
\left|\frac{1}{2} k b^{*}\left(\frac{u^{n+1}-u^{n}}{k}, e_{1}^{h, n}, \phi_{2}^{h, n+1}\right)\right| \leq \mu(h+\nu)\left\|\nabla \phi_{2}^{h, n+1}\right\|^{2} \\
+\frac{k^{2} C_{\nabla u t}^{2}}{16 \mu(h+\nu)}\left\|\nabla e_{1}^{h, n}\right\|^{2} \\
\left|\frac{1}{2} k b^{*}\left(u^{n+1}, \frac{e_{1}^{h, n+1}-e_{1}^{h, n}}{k}, \phi_{2}^{h, n+1}\right)\right| \leq \mu(h+\nu)\left\|\nabla \phi_{2}^{h, n+1}\right\|^{2} \\
+\frac{k^{2} C_{\nabla u}^{2}}{16 \mu(h+\nu)}\left\|\nabla\left(\frac{e_{1}^{h, n+1}-e_{1}^{h, n}}{k}\right)\right\|^{2}
\end{gathered}
$$


In order to obtain bounds on the third and the fourth terms, we use the error decomposition (2.0.8), triangle inequality, bound (2.0.3), regularity assumption of $u$ and Young's inequality

$$
\begin{aligned}
& \left|b^{*}\left(\phi_{2}^{h, n+1}, u_{2}^{h, n+1}, \phi_{2}^{h, n+1}\right)\right| \leq\left|b^{*}\left(\phi_{2}^{h, n+1}, u^{h, n+1}, \phi_{2}^{h, n+1}\right)\right| \\
& +\left|b^{*}\left(\phi_{2}^{h, n+1}, \eta_{2}^{n+1}, \phi_{2}^{h, n+1}\right)\right| \\
& \leq 2 \mu(h+\nu)\left\|\nabla \phi_{2}^{h, n+1}\right\|^{2} \\
& +\left\|\phi_{2}^{h, n+1}\right\|^{2}\left(\frac{C_{\nabla u}}{2}+\frac{C_{u}^{2}}{16 \mu(h+\nu)}+\frac{1}{2}\left\|\nabla \eta_{2}^{n+1}\right\|+\frac{1}{16 \mu(h+\nu)}\left\|\nabla \eta_{2}^{n+1}\right\|^{2}\right) \\
& \left|b^{*}\left(\eta_{2}^{n+1}, u_{2}^{h, n+1}, \phi_{2}^{h, n+1}\right)\right| \leq\left|b^{*}\left(\eta_{2}^{n+1}, u^{h, n+1}, \phi_{2}^{h, n+1}\right)\right|+\left|b^{*}\left(\eta_{2}^{n+1}, \eta_{2}^{n+1}, \phi_{2}^{h, n+1}\right)\right| \\
& \leq 2 \mu(h+\nu)\left\|\nabla \phi_{2}^{h, n+1}\right\|^{2} \\
& +\frac{1}{4 \mu(h+\nu)}\left\|\nabla \eta_{2}^{n+1}\right\|^{2}\left(C_{\nabla u}^{2}+\left\|\nabla \eta_{2}^{n+1}\right\|^{2}\right)
\end{aligned}
$$

For the bounds on the seventh and the eighth terms, we use the error decomposition $u_{1}^{h, n}=u^{n}-e_{1}^{h, n}$, triangle inequality, bound (2.0.4), regularity assumptions of $u$ and Young's inequality

$$
\begin{gathered}
\left|\frac{1}{2} k b^{*}\left(e_{1}^{h, n+1}, \frac{u_{1}^{h, n+1}-u_{1}^{h, n}}{k}, \phi_{2}^{h, n+1}\right)\right| \leq\left|\frac{1}{2} k b^{*}\left(e_{1}^{h, n+1}, \frac{e_{1}^{h, n+1}-e_{1}^{h, n}}{k}, \phi_{2}^{h, n+1}\right)\right| \\
+\left|\frac{1}{2} k b^{*}\left(e_{1}^{h, n+1}, \frac{u^{n+1}-u^{n}}{k}, \phi_{2}^{h, n+1}\right)\right| \leq 2 \mu(h+\nu)|| \nabla \phi_{2}^{h, n+1} \|^{2} \\
+\frac{1}{16 \mu(h+\nu)}|| \nabla e_{1}^{h, n+1}||^{2}\left(k^{2} C_{\nabla u_{t}}^{2}+k^{2}|| \nabla\left(\frac{e_{1}^{h, n+1}-e_{1}^{h, n}}{k}\right) \|^{2}\right) \\
\left|\frac{1}{2} k b^{*}\left(\frac{e_{1}^{h, n+1}-e_{1}^{h, n}}{k}, u_{1}^{h, n}, \phi_{2}^{h, n+1}\right)\right| \leq\left|\frac{1}{2} k b^{*}\left(\frac{e_{1}^{h, n+1}-e_{1}^{h, n}}{k}, u^{n}, \phi_{2}^{h, n+1}\right)\right| \\
+\left|\frac{1}{2} k b^{*}\left(\frac{e_{1}^{h, n+1}-e_{1}^{h, n}}{k}, e_{1}^{h, n}, \phi_{2}^{h, n+1}\right)\right| \leq 2 \mu(h+\nu)\left\|\nabla \phi_{2}^{h, n+1}\right\|^{2} \\
+\frac{1}{16 \mu(h+\nu)}\left\|\nabla\left(\frac{e_{1}^{h, n+1}-e_{1}^{h, n}}{k}\right)\right\|^{2}\left(k^{2} C_{\nabla u}^{2}+k^{2}\left\|\nabla e_{1}^{h, n}\right\|^{2}\right)
\end{gathered}
$$


Apply the Cauchy-Schwarz and Young's inequality to (4.2.2). Since $\left\|\nabla \cdot \phi_{2}^{h, n+1}\right\|^{2} \leq$ $d\left\|\nabla \phi_{2}^{h, n+1}\right\|^{2}$ for all $\mu>0$

$$
\begin{gathered}
\frac{\left\|\phi_{2}^{h, n+1}\right\|^{2}-\left\|\phi_{2}^{h, n}\right\|^{2}}{2 k}+(1-16 \mu)(h+\nu)\left\|\nabla \phi_{2}^{h, n+1}\right\|^{2} \\
\leq \frac{d}{4 \mu(h+\nu)} \inf _{q^{h} \in Q^{h}}\left\|\frac{p^{h, n+1}+p^{h, n}}{2}-q^{h, n+1}\right\|^{2} \\
+\frac{\nu^{2} k^{2}}{16 \mu(h+\nu)}\left\|\nabla\left(\frac{e_{1}^{h, n+1}-e_{1}^{h, n}}{k}\right)\right\|^{2} \\
+\frac{h^{2}}{4 \mu(h+\nu)}\left\|\nabla e_{1}^{h, n+1}\right\|^{2}+\frac{k^{4}}{4 \mu(h+\nu)}\left\|\rho^{n+1}\right\|_{-1}^{2} \\
+\frac{1}{4 \mu(h+\nu)}\left\|\frac{\eta_{2}^{n+1}-\eta_{2}^{n}}{k}\right\|_{-1}^{2}+\frac{h+\nu}{4 \mu}\left\|\nabla \eta_{2}^{n+1}\right\|^{2}+\frac{C_{\nabla u}^{2}}{4 \mu(h+\nu)}\left\|\nabla \eta_{2}^{n+1}\right\|^{2} \\
+\frac{k^{2} C_{\nabla u t}^{2}}{16 \mu(h+\nu)}\left\|\nabla e_{1}^{h, n}\right\|^{2}+\frac{k^{2} C_{\nabla u}^{2}}{16 \mu(h+\nu)}\left\|\nabla\left(\frac{e_{1}^{h, n+1}-e_{1}^{h, n}}{k}\right)\right\|^{2} \\
+\left\|\phi_{2}^{h, n+1}\right\|^{2}\left(\frac{C_{\nabla u}}{2}+\frac{C_{u}^{2}}{16 \mu(h+\nu)}+\frac{1}{2}\left\|\nabla \eta_{2}^{n+1}\right\|+\frac{1}{16 \mu(h+\nu)}\left\|\nabla \eta_{2}^{n+1}\right\|^{2}\right) \\
+\left.\frac{1}{4 \mu(h+\nu)}\left\|\nabla \eta_{2}^{n+1}\right\|\right|^{2}\left(C_{\nabla u}^{2}+\left\|\nabla \eta_{2}^{n+1}\right\|^{2}\right) \\
+\frac{k^{2}}{16 \mu(h+\nu)}\left\|\nabla e_{1}^{h, n+1}\right\| \|^{2}\left(C_{\nabla u_{t}}^{2}+\left\|\nabla\left(\frac{e_{1}^{h, n+1}-e_{1}^{h, n}}{k}\right)\right\|^{2}\right) \\
+\frac{k^{2}}{16 \mu(h+\nu)} \| \nabla\left(\frac{e_{1}^{h, n+1}-e_{1}^{h, n}}{k} \|\left.\right|^{2}\left(C_{\nabla u}^{2}+\left\|\nabla e_{1}^{h, n}\right\|^{2}\right)\right.
\end{gathered}
$$

Take $\mu=1 / 32$, multiply (4.2.10) by $2 k$ and sum over all time levels. It follows from the regularity assumptions of theorem that

$$
k \sum_{i=0}^{n}\left\|\rho^{i+1}\right\|_{-1}^{2} k^{4} \leq C k \sum_{i=0}^{n}\left\|\rho^{i+1}\right\|^{2} k^{4} \leq C k^{4}
$$

Therefore we obtain 


$$
\begin{array}{r}
\left\|\phi_{2}^{h, n+1}\right\|^{2}+(h+\nu) k \sum_{i=0}^{n}\left\|\nabla \phi_{2}^{h, i+1}\right\|^{2} \\
\leq \frac{C}{h+\nu} k \sum_{i=0}^{n}\left[\inf _{q^{h} \in Q^{h}}\left\|\frac{p^{h, i+1}+p^{h, i}}{2}-q^{h, i+1}\right\|^{2}\right. \\
k^{2}\left\|\nabla\left(\frac{e_{1}^{i+1}-e_{1}^{i+1}}{k}\right)\right\|^{2}+h^{2}\left\|\nabla e_{1}^{i+1}\right\|^{2}+k^{4}+\left\|\frac{\eta_{2}^{i+1}-\eta_{2}^{i}}{k}\right\|_{-1}^{2} \\
+\left\|\nabla \eta_{2}^{i+1}\right\|^{2}+k^{2}\left\|\nabla e_{1}^{i+1}\right\|^{2}+\left\|\nabla \eta_{2}^{i+1}\right\|^{4} \\
+k\left\|\nabla\left(\frac{e_{1}^{i+1}-e_{1}^{i+1}}{k}\right)\right\|^{2}\left(k\left\|\nabla e_{1}^{i+1}\right\|^{2}+k\left\|\nabla e_{1}^{i}\right\|^{2}\right) \\
+k \sum_{i=0}^{n}\left\|\phi_{2}^{h, i+1}\right\|^{2}\left[\frac{C_{\nabla u}}{2}+\frac{2 C_{u}^{2}}{(h+\nu)}+\frac{1}{2}\left\|\nabla \eta_{2}^{i+1}\right\|^{2}\right. \\
\left.+\frac{2}{h+\nu}\left\|\nabla \eta_{2}^{i+1}\right\|^{2}\right]+\left\|\phi_{2}^{h, 0}\right\|^{2}
\end{array}
$$

Take $\tilde{u}^{i}$ in the error decomposition (2.0.8) to be the $L^{2}$-projection onto $V^{h}$, for $i \geq 1$. Take $\tilde{u}^{0}$ to be $u_{0}^{s}$. This gives $\phi_{2}^{h, 0}=0$ and $e_{1}^{0}=\eta_{2}^{0}$. Also it follows from the Proposition (3.1.2) that $\left\|\eta_{2}^{0}\right\| \leq C h^{m}$; under the assumption of the theorem applying the discrete Gronwall's lemma (2.0.4) and using bounds in theorems (3.3.2), (3.3.4), give

$$
\begin{array}{r}
\left\|\phi_{2}^{h, n+1}\right\|^{2}+(h+\nu) k \sum_{i=0}^{n}\left\|\nabla \phi_{2}^{h, i+1}\right\|^{2} \\
\leq \frac{C}{h+\nu} k \sum_{i=0}^{n}\left[\inf _{q^{h} \in Q^{h}}\left\|\frac{p^{h, i+1}+p^{h, i}}{2}-q^{h, i+1}\right\|^{2}\right. \\
+\frac{k^{2}}{h+\nu}\left(h^{2}+k^{2}\right)+\frac{h^{2}}{h+\nu}\left(h^{2}+k^{2}\right)+k^{4} \\
+\left\|\frac{\eta_{2}^{i+1}-\eta_{2}^{i}}{k}\right\|_{-1}^{2}+\left\|\nabla \eta_{2}^{i+1}\right\|^{2}+\left\|\nabla \eta_{2}^{i+1}\right\|^{4} \\
\left.+\frac{k}{(h+\nu)^{2}}\left(h^{2}+k^{2}\right)\left(h^{2}+k^{2}\right)\right]+C h^{2 m}
\end{array}
$$

Use the approximation properties of $X^{h}, Q^{h}$. Since the mesh nodes do not depend upon the time level, it follows from (2.0.5), (2.0.6) that 


$$
\begin{array}{r}
k \sum_{i=0}^{n} \inf _{q^{h} \in Q^{h}}\left\|\frac{p^{h, i+1}+p^{h, i}}{2}-q^{h, i+1}\right\|^{2} \leq C h^{2 m}, \\
k \sum_{i=0}^{n}\left\|\frac{\eta_{2}^{i+1}-\eta_{2}^{i}}{k}\right\|_{-1}^{2} \leq C k \sum_{i=0}^{n}\left\|\frac{\eta_{2}^{i+1}-\eta_{2}^{i}}{k}\right\|^{2} \leq C h^{2 m}, \\
k \sum_{i=0}^{n}\left\|\eta_{2}^{i+1}\right\|^{2} \leq C h^{2 m} .
\end{array}
$$

Bounds (4.2.12) and (4.2.13) give the following result

$$
\begin{aligned}
& \left\|\phi_{2}^{h, n+1}\right\|^{2}+(h+\nu) k \sum_{i=0}^{n}\left\|\nabla \phi_{2}^{h, i+1}\right\|^{2} \\
& \leq \frac{C}{(h+\nu)^{2}}\left(h^{2 m}+h^{4}+k^{4}+h^{2} k^{2}\right) .
\end{aligned}
$$

Using the error decomposition and triangle inequality with (4.2.14), we obtain

$$
\begin{array}{r}
\left\|e_{2}^{h, n+1}\right\|+\left((h+\nu) k \sum_{i=0}^{n}\left\|\nabla e_{2}^{h, i+1}\right\|^{2}\right)^{\frac{1}{2}} \\
\leq \frac{C}{(h+\nu)}\left(h^{m}+h^{2}+k^{2}+h k\right) .
\end{array}
$$

This proves the Theorem (4.2.1). Thus, we derived the error estimates, that agree with the general theory of the defect and deferred correction methods. Briefly, the Correction Step approximation $u_{2}^{h}$ is improved by an order of $\mathrm{h}$ in space and of $\mathrm{k}$ in time, compared to the Artificial Viscosity approximation $u_{1}^{h}$.

Next, we will give some computational results. 


\section{Chapter 5}

\section{Computational Tests}

We perform one quantitative and one qualitative test of the proposed regularization procedure. In both tests the non-homogeneous Dirichlet boundary conditions are implemented, and the computational results support the theoretical findings.

\subsection{Quantitative Test}

For the quantitative assessment, consider a two-dimensional problem with a known exact solution. The traveling wave solution of the NSE in $\Omega=[0.5,1]^{2}$ is given by

$$
\begin{array}{r}
u=\left(\begin{array}{l}
0.75+0.25 \cos (2 \pi(x-t)) \sin (2 \pi(y-t)) \exp \left(-8 \pi^{2} t \nu\right) \\
0.75-0.25 \sin (2 \pi(x-t)) \cos (2 \pi(y-t)) \exp \left(-8 \pi^{2} t \nu\right)
\end{array}\right) \\
p=-\frac{1}{64}(\cos (4 \pi(x-t))+\cos (4 \pi(y-t))) \exp \left(-16 \pi^{2} t \nu\right),
\end{array}
$$

and the right-hand side $f$ and initial condition $u_{0}$ are computed so that (5.1.1) satisfies (1.0.1). The final time in the computations is taken to be $T=1$.

In order to verify the theoretical claims on the convergence rates, we take the time 
step equal to the mesh diameter, $\Delta t=h$.

For $\nu=\frac{1}{100}$ the calculated convergence rates in Tables 5.1 and 5.2 confirm what is predicted by Theorems (3.3.2) and (4.2.1) for $\left(P_{2}, P_{1}\right)$ Taylor-Hood finite elements: the convergence rates are doubled after the correction step. Notice also the asymptotic character of convergence, typical of the defect correction methods.

Table 5.1: AV approximation, $\nu=0.01$

\begin{tabular}{|c|c|c|c|c|}
\hline $\mathrm{N}$ & $\left\|u-u_{1}^{h}\right\|_{L^{2}\left(0, T ; L^{2}(\Omega)\right)}$ & rate & $\left\|u-u_{1}^{h}\right\|_{L^{2}\left(0, T ; H^{1}(\Omega)\right)}$ & rate \\
\hline 8 & 0.0139742 & - & 0.23282 & - \\
\hline 16 & 0.00945258 & 0.56 & 0.179798 & 0.37 \\
\hline 32 & 0.00580328 & 0.70 & 0.123682 & 0.54 \\
\hline 64 & 0.00331349 & 0.81 & 0.0766837 & 0.69 \\
\hline 128 & 0.00178142 & 0.90 & 0.0433087 & 0.82 \\
\hline 256 & 0.000922772 & 0.95 & 0.0228883 & 0.92 \\
\hline
\end{tabular}

Table 5.2: Correction step approximation, $\nu=0.01$.

\begin{tabular}{|c|c|c|c|c|}
\hline $\mathrm{N}$ & $\left\|u-u_{2}^{h}\right\|_{L^{2}\left(0, T ; L^{2}(\Omega)\right)}$ & rate & $\left\|u-u_{2}^{h}\right\|_{L^{2}\left(0, T ; H^{1}(\Omega)\right)}$ & rate \\
\hline 8 & 0.0106313 & - & 0.189918 & - \\
\hline 16 & 0.0060028 & 0.83 & 0.128519 & 0.56 \\
\hline 32 & 0.00272105 & 1.14 & 0.0710604 & 0.86 \\
\hline 64 & 0.000993846 & 1.45 & 0.0314236 & 1.18 \\
\hline 128 & 0.000302142 & 1.72 & 0.0111824 & 1.49 \\
\hline 256 & 0.0000817667 & 1.89 & 0.00336761 & 1.73 \\
\hline
\end{tabular}

As the viscosity coefficient $\nu$ decreases, the convergence rates improve slower - see the results for the flow at $\nu=\frac{1}{2000}$ in Tables 5.3 and 5.4 . 
Table 5.3: AV approximation, $\nu=0.0005$.

\begin{tabular}{|c|c|c|c|c|}
\hline $\mathrm{N}$ & $\left\|u-u_{1}^{h}\right\|_{L^{2}\left(0, T ; L^{2}(\Omega)\right)}$ & rate & $\left\|u-u_{1}^{h}\right\|_{L^{2}\left(0, T ; H^{1}(\Omega)\right)}$ & rate \\
\hline 8 & 0.0262208 & - & 0.439399 & - \\
\hline 16 & 0.0188948 & 0.47 & 0.367997 & 0.26 \\
\hline 32 & 0.0125722 & 0.59 & 0.291022 & 0.34 \\
\hline 64 & 0.00776946 & 0.69 & 0.2206 & 0.40 \\
\hline 128 & 0.00443914 & 0.81 & 0.159449 & 0.49 \\
\hline 256 & 0.00237518 & 0.9 & 0.108957 & 0.55 \\
\hline
\end{tabular}

Table 5.4: Correction step approximation, $\nu=0.0005$.

\begin{tabular}{|c|c|c|c|c|}
\hline $\mathrm{N}$ & $\left\|u-u_{2}^{h}\right\|_{L^{2}\left(0, T ; L^{2}(\Omega)\right)}$ & rate & $\left\|u-u_{2}^{h}\right\|_{L^{2}\left(0, T ; H^{1}(\Omega)\right)}$ & rate \\
\hline 8 & 0.0217697 & - & 0.396863 & - \\
\hline 16 & 0.0141143 & 0.63 & 0.32536 & 0.29 \\
\hline 32 & 0.00777988 & 0.86 & 0.249133 & 0.39 \\
\hline 64 & 0.0036525 & 1.09 & 0.178223 & 0.48 \\
\hline 128 & 0.00152888 & 1.26 & 0.118064 & 0.59 \\
\hline 256 & 0.000597594 & 1.36 & 0.0709845 & 0.73 \\
\hline
\end{tabular}

To further comment on the asymptotic nature of convergence of defect correction methods, notice that the a priori error estimates have the term $(h+\nu)^{-1}$ in the right hand side. This decreases the convergence rates on the coarse meshes, where $h>>\nu$. The term that contains $\nabla\left(u-u_{i}^{h}\right), i=1,2$ in the left hand side is also proportional to $(h+\nu)$, which further decreases the convergence rates in the $H^{1}$-seminorm on coarse meshes for problems with high Reynolds number. We also ran the same tests (not shown here) with $\Delta t=h^{2}$ and obtained the convergence rates very similar to those presented above, which indicates that the reduced convergence rates are due to the 
asymptotic behaviour of the defect correction, and not the deferred correction part of the error.

\subsection{Qualitative Test}

For the qualitative assessment, consider the 2-D flow past an obstacle, at high Reynolds number $R e=600$. The von Karman vortex street is expected to be seen for a fully resolved flow; on a coarse mesh with $h \sim \frac{1}{32}$ the true solution demonstrates the oscillatory behavior past the obstacle (Figure 5.1). Note that the solution is known to depend on the Reynolds number in the following manner: for $1<R e<10$ the flow is no longer symmetric behind the obstacle, for $10<R e<100$ re-circulation areas appear in the wake behind the obstacle and, as the Reynolds number grows beyond $R e=100$, these vortices develop and start to oscillate. Roughly at $R e=1000$ turbulence develops and the coherent structures in the flow disappear.

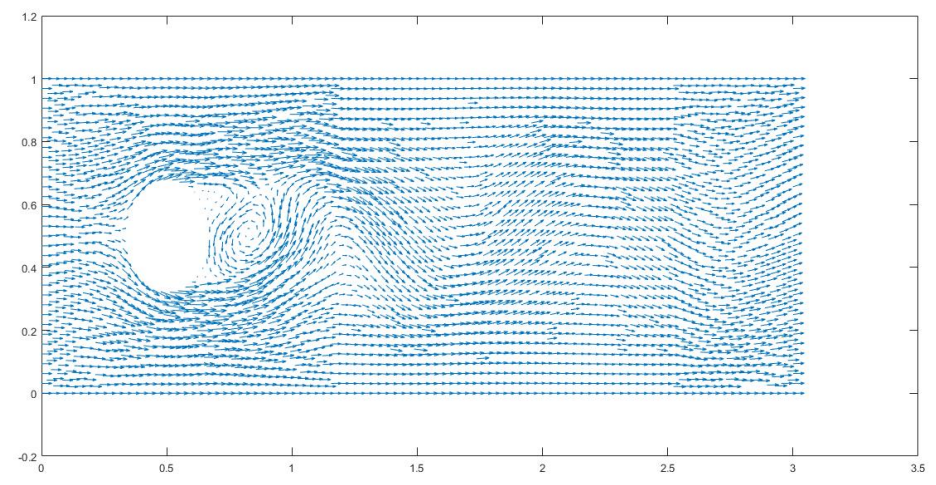

Figure 5.1: DNS velocity field $u$

We compute the defect step solution $u_{1}$ and the corrected solution $u_{2}$ on the same coarse mesh with 32 nodes per unit boundary $\left(h \sim \frac{1}{32}\right)$. The domain is $\Omega=$ 
$[0,1] \times[0,3]$ with a circle of radius 0.15 , centered at $(0.5,0.5)$, cut out of $\Omega$. The parabolic inflow on the left boundary is introduced, with zero forcing. The results were computed with $R e=600, T=20, \Delta t=h$.

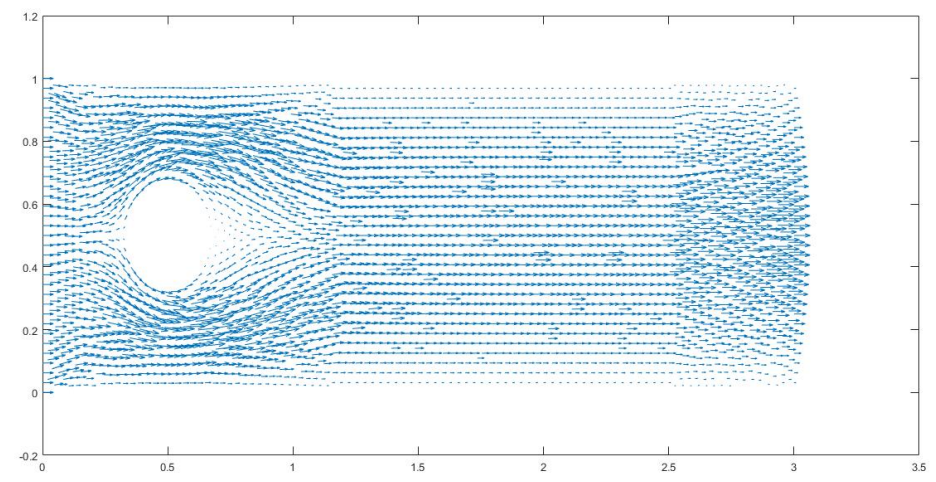

Figure 5.2: AV Approximation $u_{1}^{h}$

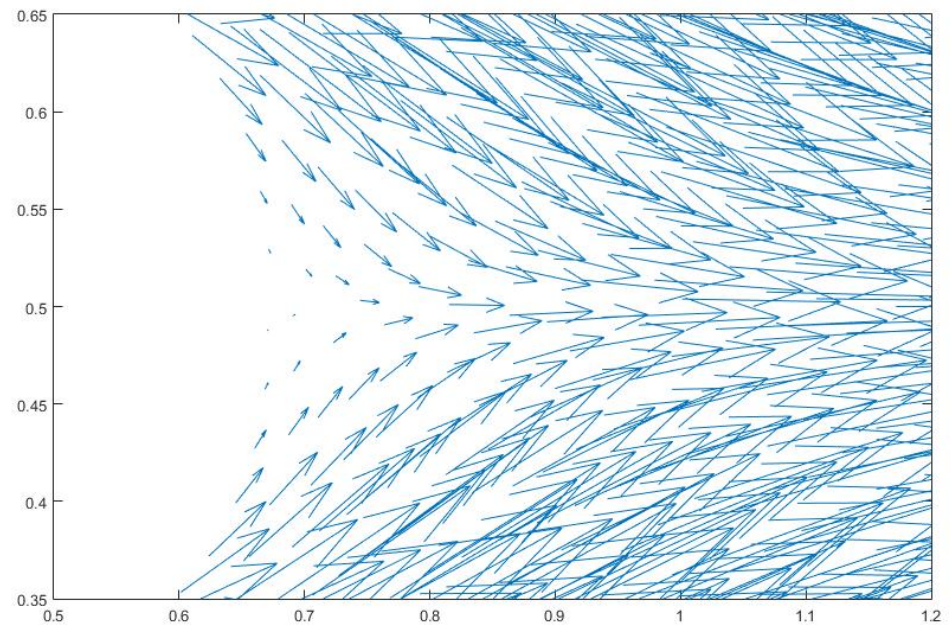

Figure 5.3: AV Approximation zoomed in

As seen in Figures 5.2 and 5.3, artificial viscosity approximation gives a result that cannot capture the flow pattern due to high viscosity coefficient and low accuracy of the AV approximation. 


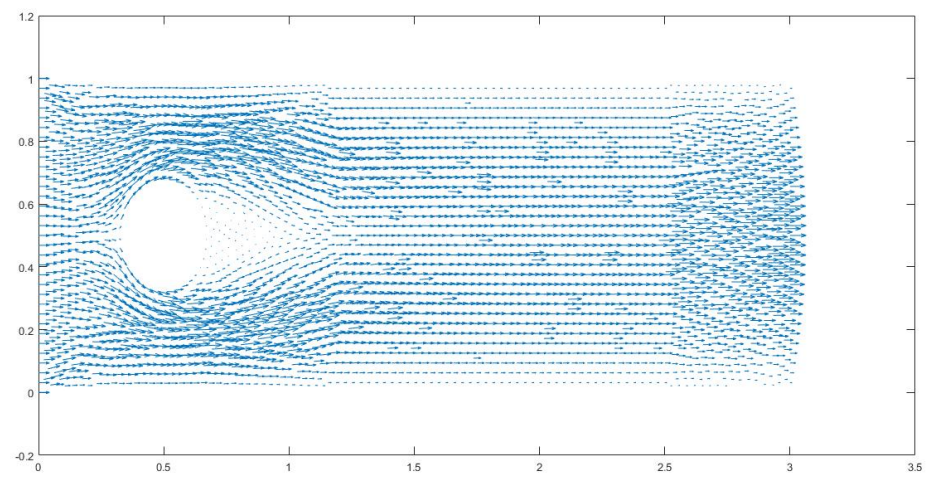

Figure 5.4: CS Approximation $u_{2}^{h}$

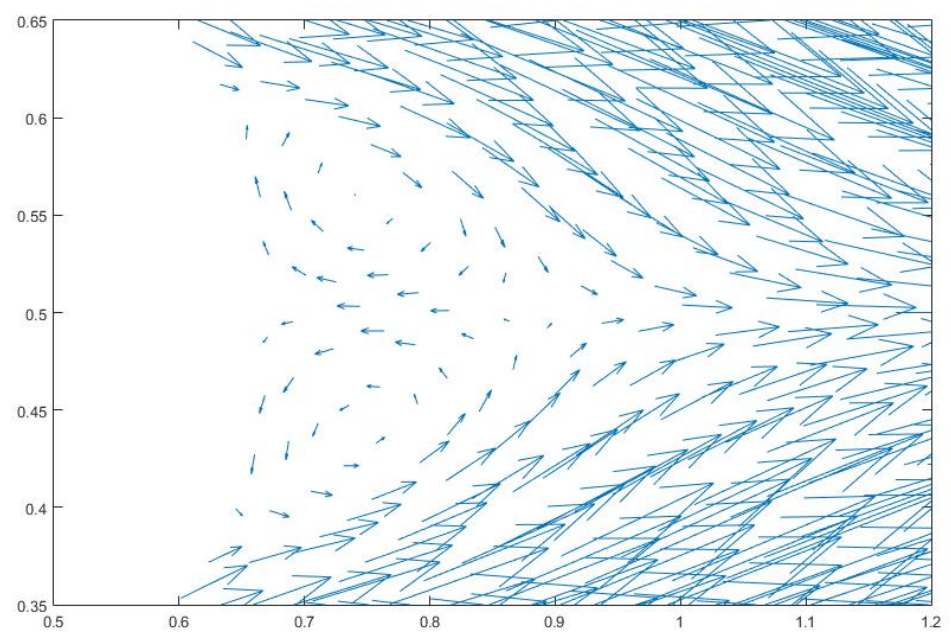

Figure 5.5: CS Approximation Zoomed in

Although the correction solution is computed with the same viscosity coefficient as the AV approximation, it gives some qualitative features of the flow pattern even on the coarse mesh - one can clearly see the re-circulation regions in the wake. This demonstrates the qualitative behavior of the correction step solution: it behaves as if the Reynolds number of the flow was increased, although the matrix of the system remains the same as in the AV case. Thus, the benefits of using the correction procedure 
are clear: for virtually no extra cost (when the parallelization is implemented) one can model turbulent flows at increasing Reynolds numbers (the interesting possibility that two or three correction steps would deepen this effect is yet to be explored). 


\section{Chapter 6}

\section{Conclusions}

The method for solving nearly singular, time-dependent problems is presented. It combines both deferred correction method for the time derivative and the defect correction method for the spatial operator. The method is applied to the NavierStokes equations, and the stability and the error estimate results for velocity is given. As observed in both theoretical and numerical results, the method presented is high accurate in both time and space. 


\section{Bibliography}

[1] K. Bohmer, P. W. Hemker, H. J. Stetter, The defect correction approach, in: K. Bohmer, H. J. Stetter (Eds.), Defect Correction Methods. Theory and Applications, Springer Verlag, 1984, pp. 1-32.

[2] P. W. Hemker, Mixed defect correction iteration for the accurate solution of the convection diffusion equation, pp. 485-501 in: Multigrid Methods, L.N.M. vol. 960, (W. Hackbusch and U. Trottenberg, eds.) Springer Verlag, Berlin 1982.

[3] P. W. Hemker, The use of defect correction for the solution of a singularly perturbed o.d.e., preprint, Mathematisch Centrum. Numerieke Wiskunde ; NW $139 / 82$

[4] O. Axelsson and W. Layton, Defect correction methods for convection dominated, convection-diffusion equations, RAIRO J. Numer. Anal. 24 (1990) pp. 423-455.

[5] O. Axelsson and W. Layton, Optimal interior estimates for the defectcorrection, finite element approach to 2-D convection-diffusion problems, ICMA report 88-116, Univ. of Pittsburgh, 1988.

[6] W. Layton, H. K. Lee, J. Peterson, A defect-correction method for the incompressible Navier-Stokes equations, Applied Mathematics and Computation, Vol. 129, Issue 1, 2002, pp. 1-19. 
[7] V. ERvin and W. Layton, High resolution minimal storage algorithms for convection dominated, convection diffusion equations, pp. 1173-1201 in: Trans. of the Fourth Army Conf. on Appl. Math. and Comp., U.S. Army Res. Office, 1987.

[8] V. Ervin and W. Layton, An analysis of a defect correction method for a model convection diffusion equation, SIAM J. Numer. Anal., 26 (1989), 169-179.

[9] V. Ervin, W. Layton, J. Maubach, Adaptive defect correction methods for viscous incompressible flow problems, SIAM J. Numer. Anal., 37 (2000), pp. 1165-1185.

[10] H. Elman, Y.-T. Shin, Iterative methods for stabilized discrete convectiondiffusion problems, IMA J. Numer. Anal., 20 (2000), pp. 333-358.

[11] W. HeinRichs, Defect correction for convection dominated flow, SIAM J. Sci. Comput., 17 (1996), 1082-1091

[12] P. Hemker, An accurate method without directional bias for the numerical solution of a 2-D elliptic singular perturbation problem, pp. 192-206 in: Theory And Applications Of Singular Perturbations, Lecture Notes in Math. 942, W. Eckhaus and E.M. de Jaeger, eds., Sprienger-Verlag, Berlin, 1982

[13] W. Layton, Introduction to the Numerical Analysis of Incompressible Viscous Flows, SIAM publications (Computational Science and Engineering Series), 2008, ISBN: 978-0-898716-57-3.

[14] M. Gunzburger, Finite element methods for viscous incompressible flows: A guide to theory, practice, and algorithms, Academic Press, Boston, 1989.

[15] A. Labovsky, A Defect Correction Method for the Time-Dependent NavierStokes Equations, Numerical Methods for Partial Differential Equations, vol.25(1), pp.1-25, 2008. 
[16] B. Koren, Multigrid and Defect-Correction for the Steady Navier-Stokes Equations, Applications to Aerodynamics, C. W. I. Tract 74, Centrum voor Wiskunde en Informatica, Amsterdam, 1991.

[17] M.-H. Lallemand, B. Koren, Iterative defect correction and multigrid accelerated explicit time stepping schemes for the steady Euler equations, SIAM Journal on Scientific Computing, vol. 14, issue 4, 1993.

[18] V. J. Ervin, H. K. LEe, Defect correction method for viscoelastic fluid flows at high Weissenberg number, Numerical Methods for Partial Differential Equations, Volume 22, Issue 1, pp. 145 - 164, 2006.

[19] V. Girault, P.A. Raviart, Finite element approximation of the Navier-Stokes equations, Lecture notes in mathematics, no. 749, Springer-Verlag, 1979.

[20] M. L. Minion, Semi-Implicit Projection Methods for Incompressible Flow based on Spectral Deferred Corrections, Appl. Numer. Math., 48(3-4), 369-387, 2004

[21] M. L. Minion, Semi-Implicit Projection Methods for Ordinary Differential Equations, Comm. Math. Sci., 1(3), 471-500, 2003.

[22] A. Bourlioux, A. T. Layton, M. L. Minion, High-Order Multi-Implicit Spectral Deferred Correction Methods for Problems of Reactive Flows, Journal of Computational Physics, Vol. 189, No. 2, pp. 651-675, 2003.

[23] W. Kress, B. Gustafsson, Deferred Correction Methods for Initial Boundary Value Problems, Journal of Scientific Computing, Springer Netherlands, Vol. 17, No. 1-4, 2002.

[24] A. N. Kolmogorov, The local structure of turbulence in incompressible viscous fluid for very large Reynolds numbers, Doklady Akademii Nauk SSSR, vol 30, p.913, 1941. 
[25] A. Dutt, L. Greengard, V. Rokhlin, Spectral deferred correction methods for ordinary differential equations, BIT 40 (2), pp. 241-266, 2000.

[26] J.F. Gibson, J. Halcrow and P. Cvitanovic, Equilibrium and travelingwave solutions of plane Couette flow, J. Fluid Mech., Vol. 638, pp. 243 - 266, 2009; arXiv:0808.3375v2.

[27] H. J. Stetter, The defect correction principle and discretization methods, Numerische Mathematik, vol. 29(4), pp. 425-443, 1978.

[28] R. Frank, W. Ueberhuber, Iterated Defect Correction for the Efficient Solution of Stiff Systems of Ordinary Differential Equations, BIT 17, 1977, pp. 146-159.

[29] V. Girault, P.A. Raviart, Finite element approximation of the Navier-Stokes equations, Lecture notes in mathematics, no. 749, Springer-Verlag, 1979.

[30] J. Heywood, R. Rannacher, Finite-element approximations of the nonstationary Navier-Stokes problem. Part 4: Error analysis for second-order time discretization, SIAM J. Numer. Anal., 2 (1990)

[31] M. Gunzburger, A. Labovsky, High Accuracy Method for Turbulent Flow Problems, M3AS: Mathematical Models and Methods in Applied Sciences, vol. $22(6), 2012$. 